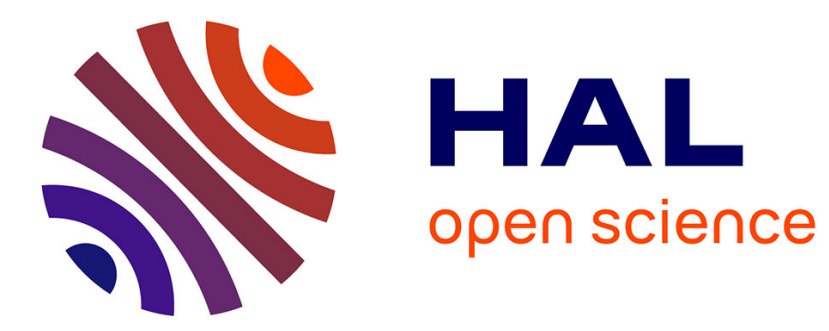

\title{
Stability analysis and non-linear behaviour of structural systems using the complex non-linear modal analysis (CNLMA)
}

\author{
Jean-Jacques Sinou, Fabrice Thouverez, Louis Jezequel
}

\section{To cite this version:}

Jean-Jacques Sinou, Fabrice Thouverez, Louis Jezequel. Stability analysis and non-linear behaviour of structural systems using the complex non-linear modal analysis (CNLMA). Computers \& Structures, 2006, 84 (29-39), pp.1891-1905. 10.1016/j.compstruc.2006.08.020 . hal-00207652

\section{HAL Id: hal-00207652 \\ https://hal.science/hal-00207652}

Submitted on 18 Jan 2008

HAL is a multi-disciplinary open access archive for the deposit and dissemination of scientific research documents, whether they are published or not. The documents may come from teaching and research institutions in France or abroad, or from public or private research centers.
L'archive ouverte pluridisciplinaire $\mathbf{H A L}$, est destinée au dépôt et à la diffusion de documents scientifiques de niveau recherche, publiés ou non, émanant des établissements d'enseignement et de recherche français ou étrangers, des laboratoires publics ou privés. 
Journal home page: http://www.sciencedirect.com/science/journal/00457949

Stability analysis and non-linear behaviour of structural systems using the complex non-linear modal analysis (CNLMA)

Computers \& Structures, Volume 84, Issues 29-30, November 2006, Pages 1891-1905

J.-J. Sinou, F. Thouverez and L. Jezequel

\title{
STABILITY ANALYSIS AND NON-LINEAR BEHAVIOUR OF STRUCTURAL SYSTEMS USING THE COMPLEX NON-LINEAR MODAL ANALYSIS (CNLMA)
}

\author{
J-J. SINOU*, F. THOUVEREZ and L. JEZEQUEL \\ Laboratoire de Tribologie et Dynamique des Systèmes UMR CNRS 5513 \\ Ecole Centrale de Lyon, 36 avenue Guy de Collongue,69134 Ecully, France.
}

\begin{abstract}
Herein, a novel non-linear procedure for producing non-linear behaviour and stable limit cycle amplitudes of non-linear systems subjected to super-critical Hopf bifurcation point is presented. This approach, called Complex Non-Linear Modal Analysis (CNLMA), makes use of the non-linear unstable mode which governs the non-linear dynamic of structural systems in unstable areas. In this study, the computational methodology of CNLMA is presented for the systematic estimation of the non-linear behavior of structural system, and it is applied to a complex system with quadratic and cubic non-linearities. Results from this non-linear method are compared with results obtained by integrating the full original non-linear system. This procedure appears very interesting in regard to computational time, formulation, and also necessitates very few computer resources. Thus the CNLMA is suited to computational implementation for various complex non-linear system with many degrees-of-freedom.
\end{abstract}

\section{KEYWORDS}

Non-linear dynamics, stability analysis, non-linear mode, friction induced vibration.

\section{INTRODUCTION}

Many complex structures which are composed of several non-linearities are subject to stable and unstable behaviour depending on physical parameters [1-8]. For such structures, estimation of the nonlinear behaviour in near-critical steady-state equilibrium points is essential. However, numerical integration may be computationally expensive. In such cases it is convenient to applied non-linear approaches by taking advantages of the dynamic properties of the non-linear dynamical systems. Most of the standard non-linear methods for generating approximate solutions of non-linear dynamical systems are based either on approaches in which the solution is represented as an assumed and approximated form [2-3,9-10] or on simplification and reduction approaches of non-linear original [1126]. 
Due to their great potential of savings in computational time for simulations, the non-linear model reduction have been studied by many researchers in recent years for weak and strong non-linear systems [14-26] and have undergone considerable evolution since their original and fundamental concept (Rosenberg [18-19]). The main objective of these non-linear methods is to extract and characterize the non-linear behaviours of structural systems by using non-linear interpretations of modes and non-linear extensions of principal coordinate transformations. This concept of non-linear normal modes has great potential for reduction techniques [20-26]. However, these non-linear methods based on reductions or simplifications are computationally intensive in the formulation stage even if they offer savings in computational time for simulations [23] (due to the reduced-order and simplified model obtained).

To avoid these disadvantages, Nayfeh and Balachandran [3, 27-28] propose in very elegant analysis to use the method of the multiples scales [3] to obtain a first-order approximation for the amplitude of the limit cycles by considering harmonic solution. They formulate a perturbation solution of the non-linear equations that is valid in the neighborhood of a given fixed point. They demonstrate that the method of multiple scales involves less algebra that the method of normal forms. As explained by Nayfeh and Balachandran , this perturbation analysis can be applied to high-dimensional systems but is restricted in the case of approximating limit cycles close to a Hopf bifurcation point. Moreover, it may be observed that the control parameter of the non-linear system needs to be expanded as a power series in a small parameter about the critical point associated with a given fixed point. In this paper, a method called the Complex Non-Linear Modal Analysis [29] that may be shown as a variant of the previous method developed by Nayfeh and Balachandran [3, 27-28], is proposed for calculating the periodic solutions of non-linear systems subjected to super-critical Hopf bifurcation point with several degrees-of-freedom. This approach is based on the evolution of the unstable mode and the estimation of the associated unstable solution curve in order to obtain an approximated periodic solution of the stable limit cycles for non-linear systems subjected to supercritical Hopf bifurcation point. The principle of the equivalent linearization based on the replacement of the non-linear dynamic system by a linear system [29-32] is applied. Using this principle, it will be possible to estimate the stable limit cycle amplitudes far from the super-critical Hopf bifurcation point. This method is tested in the case of non-linear systems with quadratic and cubic non-linearitites in order to obtain the periodic limit cycles amplitudes near and far from the Hopf bifurcation point without assuming a form for the control parameter. However, it will be shown that this CNLMA approach works in the case of stable limit cycles.

Firstly, the description and explicit construction of the Complex Non-Linear Modal Analysis for nonlinear systems is developed. Secondly, this non-linear method is applied to an illustrative example which is an complex non-linear aircraft brake system subject to friction-induced instability phenomena: results from stability analysis are briefly presented and discussed in order to detect the stable and unstable regions. In a second way, the non-linear behaviour of the system when a super-critical Hopf bifurcation point appears and the associated stable limit cycle amplitudes are approximated by using the CNLMA. Results from this non-linear modal approach are compared with results obtained by integrating the full original non-linear system. Then the suitability of the CNLMA for computational parametric design studies is demonstrated. Finally, the paper closes with a discussion concerning some of the most interesting features and results of this approach. 


\section{THE COMPLEX NON-LINEAR MODAL ANALYSIS}

\subsection{Introduction}

In the field of engineering, the description of a non-linear dynamical system is usually given by a $n$ dimensional equation

$$
\mathbf{M z}+\mathbf{C} \dot{\mathbf{z}}+\mathbf{K z}+\mathbf{F N L}(\dot{\mathbf{z}}, \mathbf{z})=\mathbf{0}
$$

where $\ddot{\mathbf{z}}, \dot{\mathbf{z}}$ and $\mathbf{z}$ are the acceleration, velocity, and displacement response $n$-dimensional vectors of the degrees-of-freedom, respectively. $\mathbf{M}$ is the mass matrix, $\mathbf{C}$ is the damping matrix, $\mathbf{K}$ is the stiffness matrix, and FNL contains the non-linear terms.

The non-linear dynamical equations are usually rewritten in state variables $\quad \mathbf{x}=\left\{\begin{array}{ll}\mathbf{z} & \dot{\mathbf{z}}\end{array}\right\}^{T}$ so that the description of dynamical systems is given by a $2 n$-dimensional differential equation

$$
\dot{\mathbf{x}}=\mathbf{F}(\mathbf{x}, \mu)
$$

where $\mu$ is a control parameter and $\mathbf{F}$ a non-linear function.

To shift the fixed point $\mathbf{x}_{\mathbf{0}}$ of the system (2) which is defined by $\mathbf{F}\left(\mathbf{x}_{\mathbf{0}}, \mu\right)=\mathbf{0}$ to the origin, the transformation $\mathbf{x}=\mathbf{y}+\mathbf{x}_{\mathbf{0}}$ is used. The non-linear system (2) is transformed by

$$
\dot{\mathbf{y}}=\mathbf{F}\left(\mathbf{x}_{\mathbf{0}}+\mathbf{y}, \mu\right)
$$

Assuming that the function $\mathbf{F}$ is at least $C^{2}$, the non-linear system (3) can be extended in a Taylor series about $\mathbf{x}_{\mathbf{0}}$ for small $\|\mathbf{y}\|$

$$
\dot{\mathbf{y}}=\mathbf{J}(\mu) \mathbf{y}+\mathbf{F}_{\mathrm{NL}}(\mathbf{y}, \mu)=\mathbf{A}(\mu) \mathbf{y}+\mathbf{F}_{2}(\mathbf{y}, \mu)+\mathbf{F}_{3}(\mathbf{y}, \mu)+\cdots+\mathbf{F}_{\mathbf{k}}(\mathbf{y}, \mu)+O\left(\mathbf{y}^{k+1}\right)
$$

where $\mathbf{J}(\mu)=\mathbf{D}_{\mathbf{x}} \mathbf{F}\left(\mathbf{x}_{\mathbf{0}}, \mu\right)$ is the $2 n \times 2 n$ matrix of first derivatives of $\mathbf{F}$ evaluated at the fixed point $\left(\mathbf{x}_{\mathbf{0}}, \mu\right)$ and $\mathbf{F}_{\mathbf{k}}(\mathbf{y}, \mu)$ defines a degree-k polynomial series in the principal coordinates of degree $\mathbf{y}$.

We remain that eigenvalues $\lambda$ of the linearized system $\mathbf{D}_{\mathbf{x}} \mathbf{F}$ provide information about the local stability of the non-linear system of (2); if all real parts of eigenvalues are inferior or egal zero, the system is stable; neither the system is unstable.

\subsection{The non-linear modal approximated solution}

Considering that the non-linear system defined in equation (3) has a super-critical Hopf bifurcation point for the control parameter $\mu=\mu_{0}$ such that

$$
\begin{aligned}
& \left.\operatorname{Re}\left(\lambda_{i}(\mu)\right)\right|_{\mathbf{x}=\mathbf{x}_{0}, \mu=\mu_{0}}=0 \\
& \left.\frac{d}{d \mu}\left(\operatorname{Re}\left(\lambda_{i}(\mu)\right)\right)\right|_{\mathbf{x}=\mathbf{x}_{0}, \mu=\mu_{0}} \neq 0 \\
& \left.\operatorname{Re}\left(\lambda_{j \neq i}(\mu)\right)\right|_{\mathbf{x}=\mathbf{x}_{0}, \mu=\mu_{0}}<0
\end{aligned}
$$

and for $\mu=\bar{\mu}+\mu_{0}>\mu_{0}$

$$
\begin{aligned}
& \left.\operatorname{Re}\left(\lambda_{i}\left(\mu=\bar{\mu}+\mu_{0}\right)\right)\right|_{\mathbf{x}=\mathbf{x}_{0}, \mu=\bar{\mu}+\mu_{0}}>0 \\
& \left.\operatorname{Re}\left(\lambda_{j \neq i}\left(\mu=\bar{\mu}+\mu_{0}\right)\right)\right|_{\mathbf{x}=\mathbf{x}_{0}, \mu=\bar{\mu}+\mu_{0}}<0
\end{aligned}
$$

the non-linear autonomous system is defined by 


$$
\dot{\mathbf{y}}=\mathbf{J}\left(\bar{\mu}+\mu_{0}\right) \mathbf{y}+\mathbf{F}_{\mathrm{NL}}\left(\mathbf{y}, \bar{\mu}+\mu_{0}\right)
$$

Assuming that all the modal participations of the stable modes (associated with the eigenvalues $\lambda_{j}\left(\bar{\mu}+\mu_{0}\right)$ ) are negligible in front of the unstable mode (associated with the eigenvalues $\lambda_{i}\left(\bar{\mu}+\mu_{0}\right)$ ), equation (3) is governed by the unstable approximated solution curve

$$
\tilde{\mathbf{y}}\left(t, \bar{\mu}+\mu_{0}\right)=\mathbf{Y}_{1}\left(\bar{\mu}+\mu_{0}\right) e^{\lambda_{1}\left(\bar{\mu}+\mu_{0}\right) t}+\mathbf{Y}_{2}\left(\bar{\mu}+\mu_{0}\right) e^{\lambda_{2}\left(\bar{\mu}+\mu_{0}\right) t}=\mathbf{Y} e^{(a+i b) t}+\overline{\mathbf{Y}} e^{(a-i b) t}
$$

where $\lambda_{1}\left(\bar{\mu}+\mu_{0}\right)=a+i b$ and $\lambda_{2}\left(\bar{\mu}+\mu_{0}\right)=a-i b$ are the complex eigenvalues of $\mathbf{D}_{\mathbf{x}} \mathbf{F}$ having a positive real part $(a>0)$ and $\mathbf{Y}$ and $\overline{\mathbf{Y}}$ define the associated eigenvectors at the unfolding parameter $\bar{\mu}+\mu_{0}$. The real part of $\lambda_{1}$ and $\lambda_{2}$ describes the behaviour of the fixed point and the imaginary part of $\lambda_{1}$ defines the value of the associated unstable frequency at the unfolding parameter $\bar{\mu}+\mu_{0}$; it may be noted that the unstable solution curve is composed of the pair of eigenvalues $\lambda_{1}$ and $\lambda_{2}$ with the associated eigenvector $\mathbf{Y}$ and conjugated eigenvector $\overline{\mathbf{Y}}$ due to the fact that the mechanical non-linear system has been rewritten in state variables.

Then, the stable periodic approximated solution (for $\mu=\bar{\mu}+\mu_{0}$ ) of the non-linear system (3), which is obtained for

$$
\operatorname{Re}\left(\lambda_{i}\right)=0 \text { for } i=1,2
$$

can be written

$$
\tilde{\mathbf{y}}(t)=\frac{p_{0}}{2}\left(\mathbf{Y}_{p_{0}} e^{i \omega_{p 0} t}+\overline{\mathbf{Y}}_{p_{0}} e^{-i \omega_{p_{0}} t}\right)
$$

where $\frac{p_{0}}{2}, \omega_{p_{0}}$ and $\mathbf{Y}_{p_{0}}$ are the amplitude, the frequency and the associated eigenvector of the periodic approximated solution governed by the unstable mode at $\bar{\mu}+\mu_{0}$. The purpose of the Complex NonLinear Modal Analysis (CNLMA) consists on the determination of these parameters $p_{0}, \omega_{p_{0}}$ and $\mathbf{Y}_{p_{0}}$ in order to obtain the periodic approximated solution $\tilde{\mathbf{y}}\left(t, p_{0}, \omega_{p_{0}}\right)$ at the unfolding parameter $\mu=\bar{\mu}+\mu_{0}$. It may be observed that no assumption is made about the value of the control parameter and more particularly that the stable limit cycle amplitudes may be estimated far from the super-critical Hopf bifurcation point.

\subsection{The Complex Non-Linear Modal Analysis (CNLMA)}

Considering the non-linear system (3) at $\mu=\bar{\mu}+\mu_{0}$, the linearized system $\dot{\mathbf{y}}=\mathbf{J}\left(\bar{\mu}+\mu_{0}\right) \mathbf{y}$ at the equilibrium point $\left(\mathbf{y}_{\mathbf{0}}=\mathbf{0}, \mu=\bar{\mu}+\mu_{0}\right)$ has one pair of unstable modes associated with the complex eigenvalues $\lambda_{1}$ and $\lambda_{2}=\bar{\lambda}_{1}\left(\operatorname{Re}\left(\lambda_{1}\right)>0\right.$ and $\left.\operatorname{Re}\left(\lambda_{2}\right)>0\right)$, and eigenvectors $\mathbf{Y}$ and $\overline{\mathbf{Y}}$.

To determine the value of $p=p_{0}$ that leads the stationary periodic approximated solution (9) of the non-linear system, the objective of the CNLMA procedure is to follow the evolution of the eigenvalues $\lambda_{i}(p)$ (with $i=1,2$ ) and the associated deformations $\mathbf{Y}_{i}(p)$ (with $i=1,2$ ) that lead to the stationary periodic solution of the system.

The evolution of the approximated solution curve $\tilde{\mathbf{y}}(t, p, \lambda)$ for a given value of $p$ is defined by

$$
\tilde{\mathbf{y}}(t, p, \lambda)=\mathbf{Y}(p) e^{\lambda(p) t}+\overline{\mathbf{Y}}(p) e^{\bar{\lambda}(p) t}
$$

with $\lambda(p)=\lambda_{1}(p)$ and $\mathbf{Y}(p)=\mathbf{Y}_{1}(p)$. As explained previously, $\operatorname{Re}(\lambda(p))$ gives information about the stability of the approximated solution (10) for each value of the value $p$. The stationary 
periodic solution and the associated value of $p_{0}$ will be obtained when $\operatorname{Re}(\lambda(p))=0$. Considering equations (5),(7), (8-9) and (10), it clearly appears that

$$
\begin{array}{lll}
\lim _{p \rightarrow 0} \operatorname{Re}(\lambda(p))=a & \text { and } & \lim _{p \rightarrow 0} \operatorname{Im}(\lambda(p))=b \\
\lim _{p \rightarrow p_{0}} \operatorname{Re}(\lambda(p))=0 & \text { and } & \lim _{p \rightarrow p_{0}} \operatorname{Im}(\lambda(p))=\omega_{p_{0}}
\end{array}
$$

Then, to follow the evolution of the unstable approximated solution curve $\tilde{\mathbf{y}}(t, p, \lambda)$, the eigenvalues $\lambda(p)$ and eigenvectors $\mathbf{Y}(p)$, the original non-linear system at the equilibrium point $\left(\mathbf{y}_{\mathbf{0}}=\mathbf{0}, \mu=\bar{\mu}+\mu_{0}\right)$ defined by

$$
\dot{\mathbf{y}}=\mathbf{J}\left(\bar{\mu}+\mu_{0}\right) \mathbf{y}+\mathbf{F}_{\mathrm{NL}}\left(\mathbf{y}, \bar{\mu}+\mu_{0}\right)
$$

will be approximated by introducing the stationary periodic solution

$$
\tilde{\mathbf{y}}\left(t, p, \omega_{p}\right)=\frac{p}{2}\left(\mathbf{Y}(p) e^{i \omega(p) t}+\overline{\mathbf{Y}}(p) e^{-i \omega(p) t}\right)=\frac{p}{2}\left(\mathbf{Y}_{p} e^{i \omega_{p} t}+\overline{\mathbf{Y}}_{p} e^{-i \omega_{p} t}\right)
$$

The non-linear approximated system is given by

$$
\dot{\tilde{\mathbf{y}}}=\mathbf{J}\left(\bar{\mu}+\mu_{0}\right) \tilde{\mathbf{y}}+\mathbf{F}_{\mathrm{NL}}\left(\tilde{\mathbf{y}}, \bar{\mu}+\mu_{0}\right)
$$

The evolutions of the eigenvalues $\lambda(p)$ and eigenvectors $\mathbf{Y}(p)$ will be calculated by considering the concept of the equivalent linearization [30-34]. This principle is based on the idea of finding a linear system which is equivalent to the non-linear system at the unstable fixed point $\left(\mathbf{x}_{0}, \bar{\mu}+\mu_{0}\right)$ :

$$
\dot{\tilde{\mathbf{y}}}=\mathbf{J}\left(\bar{\mu}+\mu_{0}\right) \tilde{\mathbf{y}}+\mathbf{J}^{\prime}\left(\bar{\mu}+\mu_{0}\right) \tilde{\mathbf{y}}=\mathbf{A}\left(\bar{\mu}+\mu_{0}\right) \tilde{\mathbf{y}}
$$

The replacement of the non-linear system (13) by a linear system is made to minimize the difference between the two systems

$$
\varepsilon=\mathbf{F}_{\mathrm{NL}}\left(\tilde{\mathbf{y}}, \bar{\mu}+\mu_{0}\right)-\mathbf{J}^{\prime}\left(\bar{\mu}+\mu_{0}\right) \tilde{\mathbf{y}}
$$

The minimization of $\boldsymbol{\varepsilon}$ is performed according to the criterion $\operatorname{Min}\left(\int_{0}^{2 \pi / \omega} \boldsymbol{\varepsilon}^{\mathrm{T}} \boldsymbol{\varepsilon}\right)$ and the procedure used to solve the optimisation problem is the least-square method. In place of the mean square criterion specified previously, other criterion can be used as well. However, a comparative study of the most commonly used criteria has shown that there is no significant superiority in terms of approximate solution accuracy of any specific criterion over the others (Iwan [32-33]).Then, the solution of the associated linear system is taken as an approximation of the original non-linear problem at the unstable fixed point $\left(\mathbf{x}_{0}, \bar{\mu}+\mu_{0}\right)$. One of the advantages of this equivalent linearization procedure is that the resulting linear problem may be solved by any convenient technique, and this approach is easily implemented. Moreover, the linearized system may be obtained for a control parameter $\bar{\mu}+\mu_{0}$ far from the super-critical Hopf bifurcation point $\mu_{0}$ so that the stable limit cycle amplitudes should be estimated for all the unstable area of the non-linear system.

The evolutions of $\lambda(p)$ and $\mathbf{Y}(p)$ are obtained by determining the eigenvalues and associated eigenvectors of $\mathbf{A}$. If all eigenvalues have their real part negative or zero, the equivalent linearized system (14) is stable and the approximated stationary periodic solution is given by $\tilde{\mathbf{y}}\left(t, p, \omega_{p}\right)=\mathbf{Y}_{p} e^{i \omega_{p} t}+\overline{\mathbf{Y}}_{p} e^{-i \omega_{p} t}$. If the previously condition is not verified, it means that the approximated solution (12) is always on the unstable approximated solution curve $\tilde{\mathbf{y}}(t, p, \lambda)$ defined in equation (10);

it implies that the value of $p$ is to small in order to reach the stable periodic solution. 
To obtain the stable approximated periodic solution $\tilde{\mathbf{y}}\left(t, p_{0}, \omega_{p_{0}}\right)$, the value $p$ is incremented by $\delta p$ and the unstable eigenvalues and eigenvectors of equation (12) are corrected by considering the new estimation of the eigenvalues $\lambda_{\text {unstable }}$ of $\mathbf{A}$ corresponding to the equivalent linearized system (14) and having their real part positive (with the associated eigenvectors $\mathbf{Y}_{\text {unstable }}$ )

$$
\begin{aligned}
& p \rightarrow p+\delta p \\
& \lambda(p) \rightarrow \lambda(p+\delta p)=\lambda_{\text {unstable }} \\
& \mathbf{Y}(p) \rightarrow \mathbf{Y}(p+\delta p)=\mathbf{Y}_{\text {unstable }}
\end{aligned}
$$

Finally, the value $p_{0}$ and the associated stable periodic approximated solution

$$
\tilde{\mathbf{y}}\left(t, p=p_{0}, \omega_{p}=\omega_{p_{0}}\right)=\frac{p_{0}}{2}\left(\mathbf{Y}\left(p=p_{0}\right) e^{i \omega\left(p=p_{0}\right) t}+\overline{\mathbf{Y}}\left(p=p_{0}\right) e^{-i \omega\left(p=p_{0}\right) t}\right)=\frac{p_{0}}{2}\left(\mathbf{Y}_{p_{0}} e^{i \omega_{p_{0}} t}+\overline{\mathbf{Y}}_{p_{0}} e^{-i \omega_{p_{0}} t}\right)
$$

are obtained by incrementing $p$ and calculating the eigenvalues of the linearized equivalent system (14) until $\operatorname{Re}\left(\lambda_{\text {unstable }}(p)\right)=0$ (with $\operatorname{Re}(\lambda(p))<0$ for the others eigenvalues of $\mathbf{A}$ ). The complete procedure and description of the Complex Non-Linear Analysis is illustrated in Figure 1.

Considering the previous procedure and associated developments, it may be shown that the CNLMA approach works only if the limit cycles are stable. Effectively, if the limit cycles are unstable, by increasing the value of the parameter $p$, the evolution of the real part of eigenvalues corresponding to the equivalent linearized system (14) and the evolution of the approximated solution curve $\tilde{\mathbf{y}}(t, p, \lambda)$ given in equation (20) may be affected by the nature of the limit cycles.

\section{APPLICATION OF THE CNLMA}

To demonstrate the non-linear procedure and ideas presented above, the Complex Non-Linear Modal Analysis is applied to a complex problem for which instability and the associated stable non-linear limit cycles have been observed.

\subsection{Non-linear dynamical system}

The system consists on a aircraft brake system as illustrated in Figure 2 : industry experience [35] has identified an important specific unstable vibration mode called whirl. Experimental approaches and laboratory vibrational tests that represent the aircraft operating environment indicate that the consequences of this whirl instability due to friction-induced vibration can be drastic and cause large amplitude oscillations of the aircraft brake system components. Whirl instability is defined as a complex rotating-bending mode of the brake and axle around $200-300 \mathrm{~Hz}$ (Feld [36]).

The whirl modelling and the complete equations of the non-linear autonomous system have been developed in a previous study [37] and are briefly described in Annexe A. This non-linear system can be written is the following form

$$
\mathbf{M} \ddot{\mathbf{x}}+\mathbf{C} \dot{\mathbf{x}}+\mathbf{K x}=\mathbf{F}_{\text {pressure }}+\mathbf{F}^{\mathrm{NL}}(\mathbf{x})
$$

where $\ddot{\mathbf{x}}, \dot{\mathbf{x}}$ and $\mathbf{x}$ are the acceleration, velocity, and displacement response 15-dimensional vectors of the degrees-of-freedom, respectively. $\mathbf{M}$ is the mass matrix, $\mathbf{C}$ is the damping matrix, $\mathbf{K}$ is the stiffness matrix, $\mathbf{F}_{\text {pressure }}$ is the vector force due to brake pressure and $\mathbf{F}^{\mathbf{N L}}$ contains the linear and non-linear quadratic and cubic terms due to the friction contact.

\subsection{Stability analysis and detection of unstable regions}

To examine the stability of the non-linear system (20) around an equilibrium point $\mathbf{x}_{\mathbf{0}}$ defined by 
the solution $\lambda$ of the characteristic equation

$$
\mathbf{K} \mathbf{x}_{0}=\mathbf{F}_{\text {pressure }}+\mathbf{F}^{\mathrm{NL}}\left(\mathbf{x}_{0}\right)
$$

$$
\operatorname{det}\left(-\lambda^{2} \mathbf{M}+\lambda \mathbf{C}+\mathbf{K}-\mathbf{K}_{\mathbf{L}}^{\mathbf{N L}}\right)=0
$$

need to be determined. $\mathbf{K}_{\mathbf{L}}^{\mathrm{NL}}$ defines the linearized expressions of the non-linear terms $\mathbf{F}^{\mathrm{NL}}$ around the equilibrium point $\mathbf{x}_{\mathbf{0}}$. The system becomes unstable if any of the eigenvalues $\lambda$ develop positive real parts.

A computational example considering various values of the friction coefficient is illustrated in Figures 3-7. Figure 3 indicates the evolution of all the real part. It appears that the system is unstable for $\mu \in\left[\begin{array}{ll}0.32 & 0.81\end{array}\right]$. Figure 4 illustrates the evolution of the two coupling unstable modes for $\mu \in[0.32$ 0.81]. The unstable mode occurs at $250 \mathrm{~Hz}$ that indicates a perfect correlation with experimental tests [35]. Finally, Figure 5 gives the evolution of the unstable mode in the complex plane with the determination of the two Hopf bifurcation points corresponding to the beginning and the end of the instability.

\subsection{Stable non-linear limit cycles}

To evaluate the vibration amplitudes at the unstable fixed point, the time-history solutions of the full set of the non-linear equations can be calculated by using a classic fourth-order Runge-Kutta algorithm. Figure 6 shows the transient response analysis and the predicted non-linear vibration amplitudes of all the displacements $\mathbf{x}$ and velocities $\dot{\mathbf{x}}$ at the instability region $\mu=\bar{\mu}+\mu_{0}=1.1 \mu_{0}$ (in this study the friction coefficient is used as the control parameter). As illustrated in the Figure 6, the non-linear oscillations $(\mathbf{x}, \dot{\mathbf{x}})$ grow until periodic oscillations called limit cycle amplitudes. Even if the timehistory oscillations have been obtained using a fourth-order Runge-Kutta algorithm, this procedure is rather expensive and consumes considerable resources both in terms of the computation time and in terms of the data storage requirements.

To avoid all these inconvenients the Complex Non-Linear Analysis will now be applied to obtain the stable periodic approximated oscillations of the non-linear dynamical system (20).

The transformation $\mathbf{x}=\mathbf{y}+\mathbf{x}_{\mathbf{0}}$ permits to shift the fixed point $\mathbf{x}_{\mathbf{0}}$ of the system (20) to the origin. In state variables $\mathbf{y}=\left\{\begin{array}{ll}\mathbf{x} & \dot{\mathbf{x}}\end{array}\right\}^{T}$ the non-linear system (20) is given by

$$
\dot{\mathbf{y}}=\mathbf{J}\left(\bar{\mu}+\mu_{0}\right) \mathbf{y}+\mathbf{F}_{\mathrm{NL}}\left(\mathbf{y}, \bar{\mu}+\mu_{0}\right)
$$

with

$$
\begin{aligned}
& \mathbf{J}=\left[\begin{array}{cc}
\mathbf{0} & \mathbf{I} \\
-\mathbf{M}^{-1}\left(\mathbf{K}-\mathbf{K}_{\mathrm{L}}^{\mathrm{NL}}\right) & -\mathbf{M}^{-1} \mathbf{C}
\end{array}\right] \\
& \mathbf{F}_{\mathrm{NL}}(\mathbf{y})=\left[\begin{array}{c}
\mathbf{0} \\
-\mathbf{M}^{-1} \mathbf{F}^{\mathrm{NL}}(\mathbf{y})
\end{array}\right]
\end{aligned}
$$

We remain that the purpose of the CNLMA is to find the stationary approximated periodic solution of the non-linear system (23) defined as follow

$$
\tilde{\mathbf{y}}\left(t, p, \omega_{p}\right)=\frac{p}{2}\left(\mathbf{Y}_{p} e^{i \omega_{p} t}+\overline{\mathbf{Y}}_{p} e^{-i \omega_{p} t}\right)
$$

by following the evolution of the real part and imaginary part of the unstable mode. 
The computational procedure described previously in Figure 1 is used; Figure 7 illustrates the evolution of the real part for the unstable mode. The final stationary approximated solution is obtained for $p_{0}=2.4$. The associated evolution of the imaginary part which describes the frequency evolution of the unstable mode is shown in Figure 8.

The approximated limit cycle amplitudes for each degree-of-freedom are given in Figure 9. It may be observed that the stationary approximated periodic solutions are closed to the non-linear solutions obtained by considering the original non-linear system (previously calculated by applying the $4^{\text {th }}$ order Runge-Kutta algorithm).

\subsection{Parametric studies for instability phenomena}

As explained in the previous section, the CNLMA procedure appears very interesting in regard to computation time and requires less computer resources in order to obtain the limit cycle amplitude.

Then this computational approach is advantageous when parametric studies are applied. In this section, we illustrate the possibility to investigate parametric studies using two parameters in order to conduct complex stability analysis and in order to determine the limit cycle amplitudes in the unstable regions.

For example, Figure 10 shows stability parametric analysis with various brake friction coefficient and various brake hydraulic pressures. In this Figure 10, the black line drawn on the map describing the real part evolution of the potential unstable eigenvalue (associated to the unstable mode) defines the contour where this real part is zero. Then, this black line represents the borderline between the stable and unstable regions versus the brake friction coefficient and the brake hydraulic pressure.

Moreover Figure 11 illustrated the associated evolutions of the frequencies in the complex plane. It may be observed that the unstable mode is in the $240-252 \mathrm{~Hz}$ frequency range.

Now, the CNLMA will be applied to obtain the limit cycle amplitudes when the fixed point is unstable. Two parametric studies will be considered. The first one considers the evolution of the stable limit cycles amplitudes versus the evolution of the friction coefficient $\mu / \mu_{\max }=\left(\bar{\mu}+\mu_{0}\right) / \mu_{\max }$ for $\mathrm{P} / \mathrm{P}_{\max }=0.5$. We remain that the fixed point unstable for $\mu / \mu_{\max } \in\left[\begin{array}{ll}0.32 & 0.81\end{array}\right]$ as indicated in Figure 3 . The evolution of value $p$ of the unstable solution (11) used in order to determine the stable periodic solution via the CNLMA is indicated in Figure 12. Figure 13 shows the evolution of the limit cycle amplitudes for each degree-of-freedom. It may be observed that the limit cycle amplitudes are calculated near or far from the super-critical Hopf bifurcation point.

Secondly, the limit cycle evolutions versus the evolution of the brake hydraulic pressure $\mathrm{P} / \mathrm{P}_{\max }$ are determined for $\mu=1.1 \mu_{0}$ as illustrated in Figure 14. The associated evolution of the Hopf bifurcation point is indicated in Table 1. Figure 15 shows the evolution of value $p$ for the unstable mode used in the CNLMA computational process. As indicated in Figure 14, the equilibrium point changes for the various brake pressure: it is clearly shown for the degrees-of-freedom $x_{S}$ and $x_{r}$.

Thus the CNLMA approach permits the determination of approximated limit cycle amplitudes of the non-linear original system near the Hopf bifurcation point (as illustrated in Figure 13) but also far from the Hopf bifurcation point (as illustrated in Figure 14). In all cases, the evolutions of the fixed point are determined.

\section{SUMMARY AND CONCLUSION}

In this paper, the Complex Non-Linear Modal analysis is presented and developed for systematic estimations of non-linear behaviour of structural systems subjected to super-critical Hopf bifurcation point. This method uses the non-linear unstable mode which governs the non-linear dynamic of 
structural systems in unstable areas in order to obtain the periodic approximated oscillations of nonlinear mechanical systems. In this study, the computational methodology of CNLMA is applied to a complex aircraft brake system with quadratic and cubic polynomial non-linear terms. Excellent agreements are found between the results obtained by the CNLMA method and the complete solution of the non-linear system integrated by using the $4^{\text {th }}$ order Runge Kutta algorithm.

Some of the most interesting features of the CNLMA method are the following: firstly, one main advantage is that this method is suited to computational implementation and appropriate to complex non-linear system with many degrees-of-freedom. Secondly, this non-linear modal approach offers savings in computational time for simulations and parametric design studies; it appears very interesting in regard to formulation stages, and also necessitates very few computer resources. Finally, this method is clearly not restricted to dynamical systems with polynomial non-linearities and may be used for various non-linear systems.

This non-linear modal method shows significant promise for the understanding of non-linear behaviour of complex non-linear dynamical system. It is hoped that this non-linear modal approach will be applied for large-scale engineering structures subject to instabilities in order to obtain rapidly approximated solutions in the unstable regions.

\section{NOMENCLATURE}

vector of displacement

vector of velocity

vector of acceleration

equilibrium point

small perturbation

damping matrix

stiffness matrix

mass matrix

M

F pressure

vector force due to the net hydraulic pressure

$F^{\mathrm{NL}}$

vector of linear and non-linear terms

$\mathbf{J}$

$\mathbf{J}^{\prime}$

$\tilde{\mathbf{y}}$

Jacobian matrix of the linear system in state variables

matrix of the equivalent linear system in state variables

approximated solution of the unstable mode

$\omega_{p} \quad$ pulsation's evolution of the unstable mode

$\mathbf{Y}_{p} \quad$ eigenvector's evolution of the unstable mode

amplitude of the unstable mode

$\omega_{p_{0}}$

pulsation of the stationary periodic approximated solution

$\mathbf{Y}_{p_{0}}$

eigenvector of the stationary periodic approximated solution

$p_{0}$

amplitude of the stationary periodic approximated solution

$\mu$

brake friction coefficient

$\bar{\mu}$

unfolding brake friction coefficient

$\mu_{0}$

brake friction coefficient at the Hopf bifurcation point 


\section{ANNEXE 1 : WHIRL MODELING}

The whirl instability due to friction-induced vibration is defined as a wobbling motion between the brake's rotating and stationary parts [36-39]: the disks in the brake stack are compressed by the hydraulic pressure applied to the brake, as illustrated in Figure 16. Without vibration, the normal pressure is distributed uniformly over the rubbed surface between rotating and stationary disks. When vibration is present, disks in the brake stack are subjected to out-of-plane rotation called accordion motion. Then, the uniform normal pressure over the disk interface is altered by this accordion notion : the normal pressure increases over half of the interface and relaxes over the other half. Mechanism of friction-induced vibration for an aircraft brake system. On some aircraft brake systems, the brake rod attaches to the brake housing in an offset, such that an angle may appear between the brake rod and the housing as illustrated in Figure 17. This offset angle which can be compared with the sprag-slip mechanism [40-43] couples the normal and tangential contact force and is one of the primary cause of instability in the whirl vibration.

To reproduce the wobbling interaction between the stationary and rotating parts, the rigid body lateral displacement and the two yaws of the stator (stationary parts) and rotor (rotating parts) are considered. For simplification, the multi-stage brake is represented by a single rotor-stator system (as illustrated in Figure 18) with the effective brake friction coefficient $\mu_{\text {total }}=2 n \mu$ where $n$ is the number of interfaces between stators and rotors.

For any point $M(r, \theta)$ on the rotor-stator equivalent interface, and by considering small displacements, the normal displacement is [37-38]

$$
x(r, \theta)=x_{\text {stator }}(r, \theta)-x_{\text {rotor }}(r, \theta)=\left(x_{s}-x_{r}\right)-r \sin \theta\left(\theta_{s}-\theta_{r}\right)-r \cos \theta\left(\psi_{s}-\psi_{r}\right)
$$

where $x_{s}, x_{r}, \theta_{s}, \theta_{r}, \psi_{s}$ and $\psi_{r}$ are the stator and the rotor lateral displacement, and the stator and rotor rotations, respectively.

The normal force $F_{X}$, the moments $M_{X}, M_{Y}$ and $M_{Z}$ that generate whirl instability due to the normal contact $N(r, \theta)$ between the rotor and the stator friction surface are given by

$$
\begin{gathered}
F_{X}=\int_{0}^{2 \pi} \int_{R i}^{R 0} N(r, \theta) r d r d \theta \\
M_{X}=\int_{0}^{2 \pi} \int_{R i}^{R 0} T(r, \theta) r^{2} d r d \theta=\int_{0}^{2 \pi} \int_{R i}^{R 0} \mu_{\text {total }} N(r, \theta) r^{2} d r d \theta \\
M_{Y}=-\int_{0}^{2 \pi} \int_{R i}^{R 0} N(r, \theta) r^{2} \sin \theta d r d \theta \\
M_{Z}=-\int_{0}^{2 \pi} \int_{R i}^{R 0} N(r, \theta) r^{2} \cos \theta d r d \theta
\end{gathered}
$$

where tangential contact $T$ is generated by the Coulomb law $T(r, \theta)=\mu_{\text {total }} N(r, \theta)$.

Considering static tests [35], it has been observed that the normal contact $N(r, \theta)$ at the rotor-stator interface can be represented by a cubic polynomial in the relative displacement between the rotor and stator in compression

$$
N(r, \theta)=\sum_{i=1}^{3} K_{i} x(r, \theta)
$$


Replacing equation (32) in equations (28-31), The expressions of the normal force $F_{X}$, the moments $M_{X}, M_{Y}$ and $M_{Z}$ are given by

$$
\begin{aligned}
F_{X}= & K_{1} A_{2}\left(x_{s}-x_{r}\right)+K_{2}\left(A_{2}\left(x_{s}-x_{r}\right)^{2}+\frac{1}{4} A_{4}\left(\theta_{s}-\theta_{r}\right)^{2}+\frac{1}{4} A_{4}\left(\psi_{r}-\psi_{s}\right)^{2}\right) \\
+ & K_{3}\left(A_{2}\left(x_{s}-x_{r}\right)^{3}+\frac{3}{4} A_{4}\left(\theta_{s}-\theta_{r}\right)^{2}\left(x_{s}-x_{r}\right)+\frac{3}{4} A_{4}\left(\psi_{s}-\psi_{r}\right)^{2}\left(x_{s}-x_{r}\right)\right) \\
M_{Y}= & -\frac{1}{4} K_{1}\left(\theta_{s}-\theta_{r}\right)-\frac{1}{2} K_{2} A_{4}\left(\theta_{s}-\theta_{r}\right)\left(x_{s}-x_{r}\right) \\
& -K_{3}\left(\frac{3}{4} A_{4}\left(\theta_{s}-\theta_{r}\right)\left(x_{s}-x_{r}\right)^{2}+\frac{1}{8} A_{6}\left(\theta_{s}-\theta_{r}\right)^{3}+\frac{1}{8} A_{6}\left(\theta_{s}-\theta_{r}\right)\left(\psi_{s}-\psi_{r}\right)^{2}\right) \\
M_{X}= & \mu_{\text {total }}\left(\frac{2}{3} K_{1} A_{3}\left(x_{s}-x_{r}\right)+K_{2}\left(\frac{2}{3} A_{3}\left(x_{s}-x_{r}\right)^{2}+\frac{1}{5} A_{5}\left(\theta_{s}-\theta_{r}\right)^{2}+\frac{1}{5} A_{5}\left(\psi_{s}-\psi_{r}\right)^{2}\right)\right. \\
+ & \left.K_{3}\left(\frac{2}{3} A_{3}\left(x_{s}-x_{r}\right)^{3}+\frac{3}{5} A_{5}\left(x_{s}-x_{r}\right)\left(\theta_{s}-\theta_{r}\right)^{2}+\frac{3}{5} A_{5}\left(x_{s}-x_{r}\right)\left(\psi_{s}-\psi_{r}\right)^{2}\right)\right) \\
M_{Z}= & -\frac{1}{4} K_{1} A_{4}\left(\psi_{s}-\psi_{r}\right)-\frac{1}{2} K_{2} A_{4}\left(\psi_{s}-\psi_{r}\right)\left(x_{s}-x_{r}\right) \\
& -K_{3}\left(\frac{3}{4} A_{4}\left(\psi_{s}-\psi_{r}\right)\left(x_{s}-x_{r}\right)^{2}+\frac{1}{8} A_{6}\left(\psi_{s}-\psi_{r}\right)^{3}+\frac{1}{8} A_{6}\left(\psi_{s}-\psi_{r}\right)\left(\theta_{s}-\theta_{r}\right)^{2}\right)
\end{aligned}
$$

with $A_{k}=\pi\left(R_{0}{ }^{k}-R_{i}{ }^{k}\right) \quad$ for $2 \leq k \leq 6 . R_{0}$ and $R_{i}$ are the outer and inner radius of the discs.

Finally, the equations of motion for the non-linear aircraft brake system are

$$
\begin{aligned}
& m_{s} \ddot{x}_{s}+C_{x s} \dot{x}_{s}=F_{\text {couple } / X}+F_{h y d / X}-F_{X} \\
& I_{\theta s} \ddot{\theta}_{s}+C_{a s}\left(\dot{\theta}_{s}-\dot{\theta}_{a}\right)+K_{\theta s} \theta_{s}+K_{a s}\left(\theta_{s}-\theta_{a}\right)=F_{\text {couple } / X} R_{e}+F_{\text {couple } / Z} d_{e}+M_{Y} \\
& I_{\psi s} \ddot{\psi}_{s}+C_{a s}\left(\dot{\psi}_{s}-\dot{\psi}_{a}\right)+K_{\psi s} \psi_{s}+K_{a s}\left(\psi_{s}-\psi_{a}\right)=F_{\text {couple } / Y} d_{e}+M_{Z} \\
& I_{\varphi s} \ddot{\varphi}_{s}+C_{\varphi s} \dot{\varphi}_{s}=-F_{\text {couple } / Y} R_{e}+M_{X} \\
& m_{r} \ddot{x}_{r}+C_{x r} \dot{x}_{r}+K_{r r} X_{r}=F_{X} \\
& I_{\theta r} \ddot{\theta}_{r}+C_{b r}\left(\dot{\theta}_{r}-\dot{\theta}_{t}\right)+K_{b r}\left(\theta_{r}-\theta_{t}\right)=-M_{Y} \\
& I_{\psi r} \ddot{\psi}_{r}+C_{t r}\left(\dot{\psi}_{r}-\dot{\psi}_{t}\right)+K_{t r}\left(\psi_{r}-\psi_{t}\right)=-M_{z} \\
& m_{t} \ddot{y}_{t}+C_{t 11} \dot{y}_{t}+C_{y f t}\left(\dot{y}_{t}-\dot{y}_{f}\right)+K_{t 11} y_{t}+K_{t 12} \theta_{t}+K_{y f t}\left(y_{t}-y_{f}\right)=0 \\
& I_{t} \ddot{\theta}_{t}+C_{t 22} \dot{\theta}_{t}+C_{t r}\left(\dot{\theta}_{t}-\dot{\theta}_{r}\right)+C_{\theta f t}\left(\dot{\theta}_{t}-\dot{\theta}_{f}\right)+K_{t 21} y_{t}+K_{t 22} \theta_{t}+K_{t r}\left(\theta_{t}-\theta_{r}\right)+K_{\theta f t}\left(\theta_{t}-\theta_{f}\right)=0 \\
& m_{t} \ddot{z}_{t}+C_{t 11} \dot{z}_{t}+C_{z f t}\left(\dot{z}_{t}-\dot{z}_{f}\right)+K_{t 11} z_{t}+K_{t 12} \psi_{t}+K_{z f t}\left(z_{t}-z_{f}\right)=0 \\
& I_{t} \ddot{\psi}_{t}+C_{t 22} \dot{\psi}_{t}+C_{t r}\left(\dot{\psi}_{t}-\dot{\psi}_{r}\right)+C_{\psi f t}\left(\dot{\psi}_{t}-\dot{\psi}_{f}\right)+K_{t 21} z_{t}+K_{t 22} \psi_{t}+K_{t r}\left(\psi_{t}-\psi_{r}\right)+K_{\psi f t}\left(\psi_{t}-\psi_{f}\right) \\
& m_{f} \ddot{y}_{f}+C_{f 11} \dot{y}_{f}+C_{y f}\left(\dot{y}_{f}-\dot{y}_{t}\right)+K_{f 11} y_{f}+K_{f 12} \theta_{f}+K_{y f t}\left(y_{f}-y_{t}\right)=0 \\
& I_{f} \ddot{\theta}_{f}+C_{f 22} \dot{\theta}_{f}+C_{f s}\left(\dot{\theta}_{f}-\dot{\theta}_{s}\right)+C_{\theta f t}\left(\dot{\theta}_{f}-\dot{\theta}_{t}\right)+K_{f 21} y_{f}+K_{f 22} \theta_{f}+K_{f s}\left(\theta_{f}-\theta_{s}\right)+K_{\theta f t}\left(\theta_{f}-\theta_{t}\right)=0 \\
& m_{f} \ddot{z}_{f}+C_{f 11} \dot{z}_{f}+C_{z f t}\left(\dot{z}_{f}-\dot{z}_{t}\right)+K_{f 11} z_{f}+K_{f 12} \psi_{f}+K_{z f t}\left(z_{f}-z_{t}\right)=0 \\
& I_{f} \ddot{\psi}_{f}+C_{f 22} \dot{\psi}_{f}+C_{f s}\left(\dot{\psi}_{f}-\dot{\psi}_{s}\right)+C_{\psi f t}\left(\dot{\psi}_{f}-\dot{\psi}_{t}\right)+K_{f 21} z_{f}+K_{f 22} \psi_{f}+K_{f s}\left(\psi_{f}-\psi_{s}\right)+K_{\psi f t}\left(\psi_{f}-\psi_{t}\right)=0(51
\end{aligned}
$$

where $x_{s}, x_{r}, \theta_{s}, \theta_{r}, \psi_{s}, \psi_{r}, \varphi_{s}, y_{t}, z_{t}, \theta_{t}, \psi_{t}, y_{f}, z_{f}, \theta_{f}$ and $\psi_{f}$ are the stator and the rotor lateral displacement, the stator and rotor rotations, the piston torsional rotation and the axle deflections 
and rotations of the stator and rotor shaft, respectively. $K_{f s}$ and $C_{f s}$ define the stiffness and the damping between the stator and the shaft of the stator, called torque tube, via notches on the inner perimeter of the disk. $K_{b r}$ and $C_{b r}$ define the stiffness and the damping between the rotor and the shaft of the rotor, via drive keys on the outside of the disk. $K_{\psi f t}, K_{\theta f t}, K_{y f t}, K_{z f t}$ and $C_{\psi f t}, C_{\theta f t}, C_{y f t}, C_{z f t}$ represent the contact stiffness and the contact damping between the rotor's and stator's shaft, respectively. $K_{r r}$ represents the stiffness of the backplate of the brake. $K_{f i j}(i, j=1,2)$ and $C_{f i j}(i, j=1,2)$ are the axle bend stiffness and axle bend damping for the stator's shaft, respectively. $K_{t i j}(i, j=1,2)$ and $C_{t i j} \quad(i, j=1,2)$ are the axle bend stiffness and axle bend damping for the rotor's shaft, respectively. $d_{e}$ and $R_{e}$ represent the brake rod lateral offset and the distance axle to brake rod axis. $F_{X}, M_{X}, M_{Y}$ and $M_{Z}$ are the normal contact between the rotor and the stator friction surfaces and the associated moments calculated previously. $F_{\text {couple/X }}, F_{\text {couple/Y }}$ and $F_{\text {couple/Z }}$ represent the load due to the brake rod and are approximated by [37]

$$
\left\{\begin{array}{l}
F_{\text {couple } / X}=K_{\text {rod }} R_{e} \phi_{s} \sin \alpha+K_{\text {rod }} x_{s} \sin \alpha+K_{\text {rod }} R_{e} \theta_{s} \sin \alpha \\
F_{\text {couple } / Y}=K_{\text {rod }} R_{e} \phi_{s} \cos \alpha-K_{\text {rod }} d_{e} \theta_{s} \cos \alpha \\
F_{\text {couple } / Z}=K_{\text {rod }} R_{e} \phi_{s} \cos \alpha-K_{\text {rod }} d_{e} \psi_{s} \cos \alpha
\end{array}\right.
$$

where $K_{\text {rod }}$ defines the axial stiffness of the brake rod and $\alpha$ the sprag-slip angle due to the brake rod angle offset with the rotor/stator interface.

$F_{\text {hyd } / X}$ is the brake force due to the hydraulic pressure. It is given by is given by

$$
F_{\text {hyd/X }}=P_{\text {hydraulic }} n_{\text {piston }} \frac{\left(R_{\text {piston/outer }}^{2}-R_{\text {piston/inner }}^{2}\right)}{\left(R_{0}^{2}-R_{i}^{2}\right)}
$$

where $n_{\text {piston }}, R_{\text {piston/outer }}, R_{\text {piston/inner }}$ are the number of pistons, the outer and inner radius of the piston surface in contact with the stator, respectively. $R_{0}$ and $R_{i}$ define the outer and inner radius of the rotor/stator interface, respectively.

Finally, This non-linear 15-degree-of-freedom system has the form :

$$
\mathbf{M} \ddot{\mathbf{x}}+\mathbf{C} \dot{\mathbf{x}}+\tilde{\mathbf{K}} \mathbf{x}=\mathbf{F}_{\text {pressure }}+\mathbf{F}_{\text {couple }}(\mathbf{x})+\mathbf{F}^{\mathrm{NL}}(\mathbf{x})
$$

where $\ddot{\mathbf{x}}, \dot{\mathbf{x}}$ and $\mathbf{x}$ are the acceleration, velocity, and displacement response 15-dimensional vectors of the degrees-of-freedom, respectively. $\mathbf{M}$ is the mass matrix, $\mathbf{C}$ is the damping matrix and $\tilde{\mathbf{K}}$ is the stiffness matrix. $\mathbf{F}_{\text {pressure }}$ is the vector force due to net brake hydraulic pressure. $\mathbf{F}^{\mathbf{N L}}$ contains the linear and non-linear contact force terms at the stator and rotor interface and $\mathbf{F}_{\text {couple }}$ define the brake rod load, respectively.

Finally, the general form of the equation of motion for the non-linear system can be expressed in the following way:

$$
\mathbf{M} \ddot{\mathbf{x}}+\mathbf{C} \dot{\mathbf{x}}+(\tilde{\mathbf{K}}-\hat{\mathbf{K}}) \mathbf{x}=\mathbf{F}_{\text {pressure }}+\mathbf{F}^{\mathrm{NL}}(\mathbf{x})
$$

with $\mathbf{F}_{\text {couple }}(\mathbf{x})=\hat{\mathbf{K}} \mathbf{x}$. Finally, the non-linear 15-degree-of-freedom whirl system has the form

$$
\mathbf{M} \ddot{\mathbf{x}}+\mathbf{C} \dot{\mathbf{x}}+\mathbf{K x}=\mathbf{F}_{\text {pressure }}+\mathbf{F}^{\mathrm{NL}}(\mathbf{x})
$$

where $\ddot{\mathbf{x}}, \dot{\mathbf{x}}$ and $\mathbf{x}$ are the acceleration, velocity, and displacement response 15-dimensional vectors of the degrees-of-freedom, respectively. $\mathbf{M}$ is the mass matrix, $\mathbf{C}$ is the damping matrix and $\mathbf{K}$ is the 
global stiffness matrix. $\mathbf{F}_{\text {pressure }}$ is the vector force due to brake command and $\mathbf{F}^{\mathbf{N L}}$ contains moreover the quadratic and cubic non-linear terms.

\section{REFERENCES}

1 Guckenheimer J, Holmes P. Nonlinear oscillations, dynamical systems, and bifurcations of vector fields. Springer-Verlag, 1986.

2 Nayfeh AH, Mook DT. Nonlinear oscillations. John Wiley \& Sons, 1995.

3 Nayfeh AH, Balachandran B. Applied nonlinear dynamics : analytical, comptational and experimental methods. John Wiley \& Sons, 1995.

4 Marsden JE, McCracken M. The Hopf bifurcation and its applications. Applied Mathematical Sciences. Spring-Verlag, 1976.

5 Sinou JJ, Thouverez F, Jézéquel L. Methods to reduce non-Linear mechanical systems for instability computation. Archives of Computational Methods in Engineering: State of the Art Reviews. 2004; 11(3), 255-342.

6 Hsu L. Analysis of critical and post-critical behaviour of non-linear dynamical systems by the normal form method, part I : Normalisation formulae. Journal of Sound and Vibration. 1983; 89, 169-181.

7 Hsu L. Analysis of critical and post-critical behaviour of non-linear dynamical systems by the normal form method, part II : Divergence and flutter. Journal of Sound and Vibration. 1983; 89, 183-194.

8 Sinou JJ, Thouverez F, Jézéquel L. Analysis of friction and instability by the center manifold theory for a non-linear sprag-slip model. Journal of Sound and Vibration. 2003; 527-559.

9 Cameron TM, Griffin JH. An alternating frequency/time domain method for calculating the steady state response of nonlinear dynamic. Journal of Applied Mechanics. 1989; 56, 149-154.

10 Pierre C, Ferri AA, Dowell EH. Multi-harmonic analysis of dry friction damped systems using an incremental harmonic balance method. Journal of Applied Mechanics. 1985; 52, 958-964.

11 Baker GA, Graves-Morris P. Cambridge University Press, Padé Approximants, 1996.

12 Brezinski C. Extrapolation algorithms and Padé approximations: a historical survey. Applied Numerical Mathematics. 1983; 20, 299-318.

13 Hughes Jones R, Makinson GJ. The generation of Chisholm rational approximants to power series in two variables. Journal. Inst. math. Appl. 1974; 13, 299-310.

14 Carr J. Application of center manifold. Springer-Verlag, 1981.

15 Jézéquel L, Lamarque $\mathrm{CH}$. Analysis of non-linear dynamical systems by the normal form theory. Journal of Sound and Vibration. 1991; 149, 429-459.

16 Yu P. Computation of normal forms via a perturbation technique. Journal of Sound and Vibration. 1998; 211, 19-38.

17 Sinou JJ, Thouverez F, Jézéquel L. Extension of the center manifold approach, using the rational fractional approximants, applied to non-linear stability analysis. Nonlinear Dynamics. 2003; 33: 267-282.

18 Rosenberg RM. The normal modes of nonlinear n-degree-of-freedom systems. Journal of Applied Mechanics. 1962; 82, 7-14.

19 Rosenberg RM. On non-linear vibrations of systems with many degree of freedom. Advances in Applied Mechanics. 1966; 9, 155-242.

20 Szemplinska-Stupinicka W. The modified single mode method in the investigation of the resonant vibration of non-linear systems. Journal of Sound and Vibration. 1979; 104(2), 475-489. 
21 Szemplinska-Stupinicka W. The behaviour of nonlinear vibrating systems. Kluwer Academic, 1990.

22 Shaw SW, Pierre C. Normal modes for non-linear vibratory systems. Journal of Sound and Vibration. 1993; 164(1), 85-124.

23 Shaw SW, Pierre C, Pesheck E. Modal analysis-based reduced-order models for nonlinear structures-an invariant manifold approach. The Shock and Vibration Digest. 1999; 31(1), 3-16.

24 Shaw SW, Pierre C. Non-Linear Normal Modes and Invariant Manifolds. Journal of Sound and Vibration. 1991; 150(1), 170-173.

25 Vakakis AF, Manevitch LI, Mikhlin YV, Andpilipchuck VN, Zevin AA. Normal modes and localization in nonlinear systems. John Wiley \& Sons, 1996.

26 Vakakis AF., Non-linear normal modes and their applications in vibration theory : an overview. Mechanical System Signal Processing. 1997; 11, 3-22.

27 Nayfeh AH, Balachandran B., Cyclic motions near a Hopf bifurcation of a four-dimensional system. Nonlinear Non-linear dynamics, 1992, 1, 19-39.

28 Nayfeh AH, Balachandran B., Motion near a Hopf bifurcation of a three-dimensional system. Mechanics Research Communications, 1990, 17(4), 191-198.

29 Sinou JJ, Thouverez F, Jézéquel L. Application of a nonlinear modal instability approach to brake systems. Journal of Vibration and Acoustics. 2004; 126(1), 101-107.

30 Bogoliubov N, Mitropolski A. Asymptotic methods in the theory of nonlinear oscillations. Gordon and Breach, New York, 1961.

31 Spanos PTD, Iwan WD. On the existence and uniqueness of solutions generated by equivalent linearization. International Journal of Non-Linear mechanics. 1978; 13, 71-78.

32 Iwan WD, Patula FJ. The merit of different minimization criteria in approximate analysis. Journal of Applied Mechanics. 1972; 39, 257-262.

33 Iwan WD. A generalization of the concept of equivalent linearization International Journal of Nonlinear Mechanics. 1973; 8, 279-287.

34 Spanos PTD, Iwan WD. Harmonic analysis of dynamic systems with nonsymmetric nonlinearities, Journal of Dynamic Systems, Measurement, and Control. 1979; 101, 31-36.

35 Sinou JJ, Thouverez F, Dereure O, Mazet GB. Non-linear dynamics of a complex aircraft brake system. Experimental and theorical approaches. ASME Design Engineering Technical Conferences, 2003.

36 Feld DJ, Fehr DJ. Complex eigenvalue analysis applied to an aircraft brake vibration problem. ASME Design Engineering Technical Conferences, 1995.

37 Sinou JJ. Synthèse non-linéaire des systèmes vibrants. Application aux système de freinage. $\mathrm{PhD}$ thesis n 2002-23, Ecole Centrale de Lyon, France, 2002.

38 Liu SY, Ozbek MA, Gordon JT. A nonlinear model for aircraft brake squeal analysis. Part i : model description and solution methodology. ASME Design Engineering Technical Conferences, 1996.

39 Liu SY, Ozbek MA, Gordon JT. A nonlinear model for aircraft brake squeal analysis. Part ii : stability analysis and parametric studies. ASME Design Engineering Technical Conferences, 1996.

40 Ibrahim RA. Friction-induced vibration, chatter, squeal and chaos : part I - mechanics of contact and friction. Applied Mechanics Review. 1994; 47(7), 209-226.

41 Ibrahim RA. Friction-induced vibration, chatter, squeal and chaos : part II - dynamics and modeling. Applied Mechanics Review. 1994; 47(7), 227-253

42 Spurr RT. A theory of brake squeal. Proc. Auto. Div. Instn. Mech. Engrs. 1961; 33-40.

43 Oden JT, Martins JAC. Models and computational methods for dynamic friction phenomena. Computer Methods in Apllied Mechanics and Engineering. 1985; 52, 527-634. 


\begin{tabular}{cc}
\hline Pressure $\mathrm{P} / \mathrm{P}_{\max }$ & Hopf bifurcation point $\mu_{0} / \mu_{\max }$ \\
\hline 0.1667 & 0.1458 \\
0.25 & 0.2061 \\
0.3333 & 0.2618 \\
0.4167 & 0.303 \\
0.5 & 0.3323 \\
0.5833 & 0.3538 \\
0.6667 & 0.3697 \\
0.75 & 0.3822 \\
0.8333 & 0.3920 \\
0.9167 & 0.4 \\
1 & 0.4067 \\
\hline
\end{tabular}

Table 1 : evolution of the Hopf bifurcation point $\mu_{0} / \mu_{\max }$ for various brake pressures $\mathrm{P} / \mathrm{P}_{\max }$ 


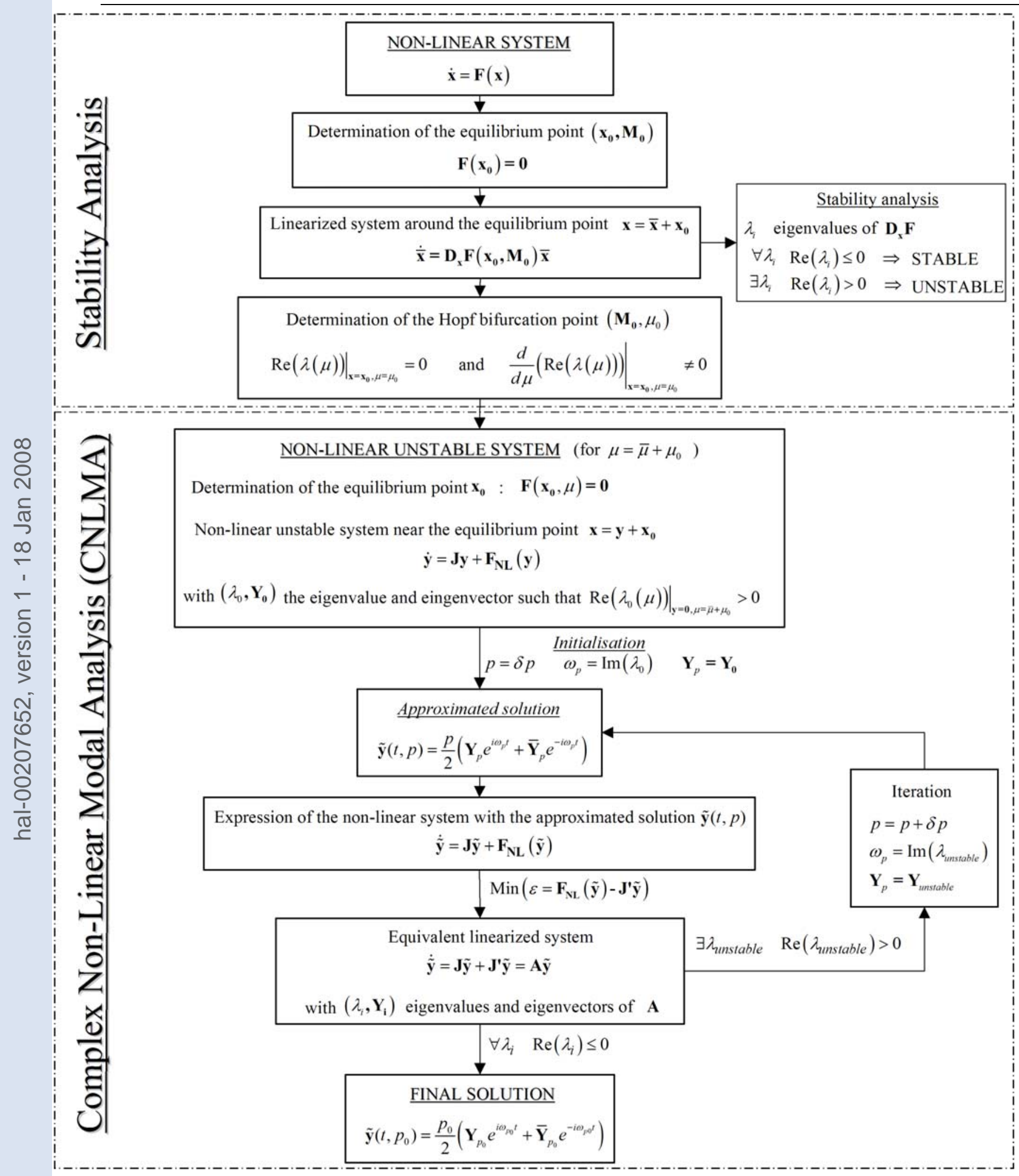

Figure 1 : Computational procedure of the CNLMA 


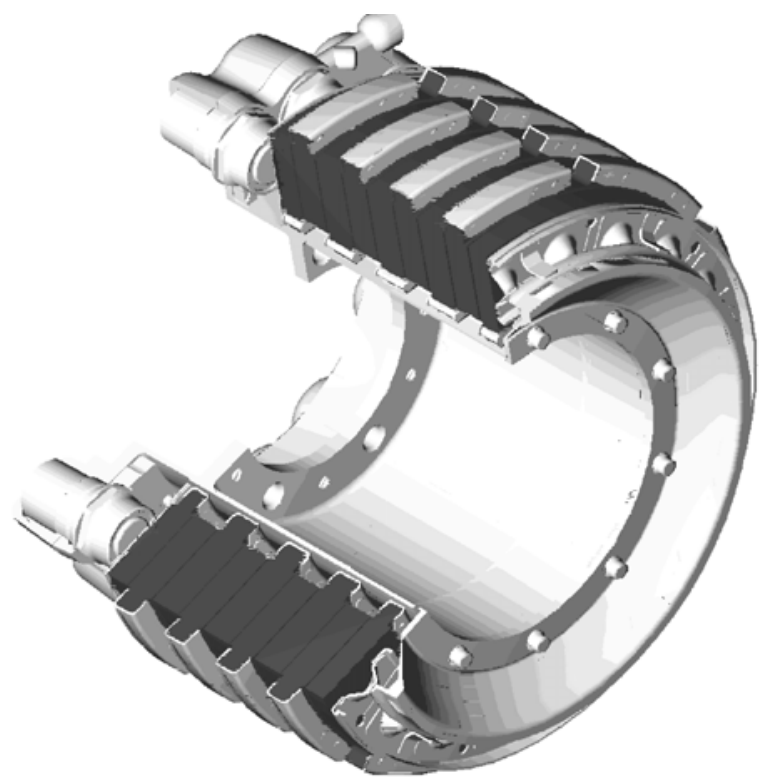

Figure 2 : aircraft brake system

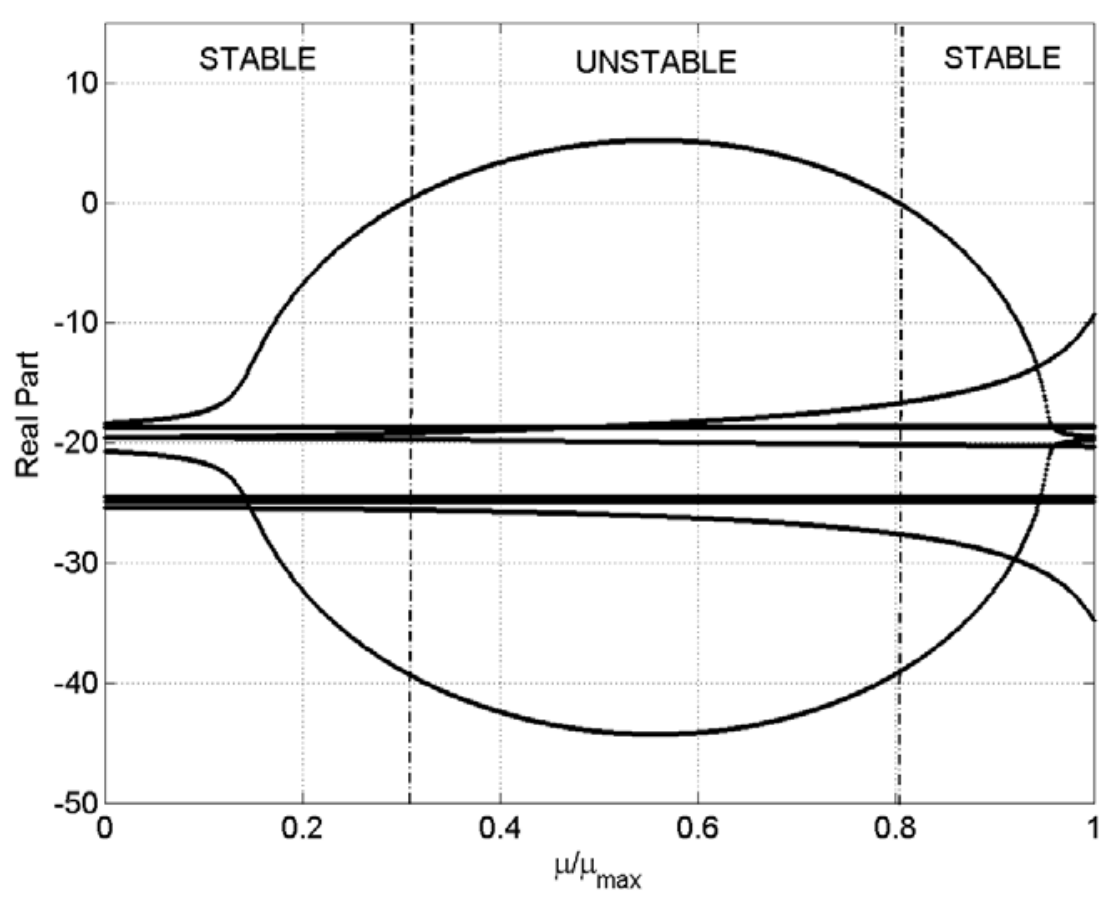

Figure 3 : evolution of the real part 


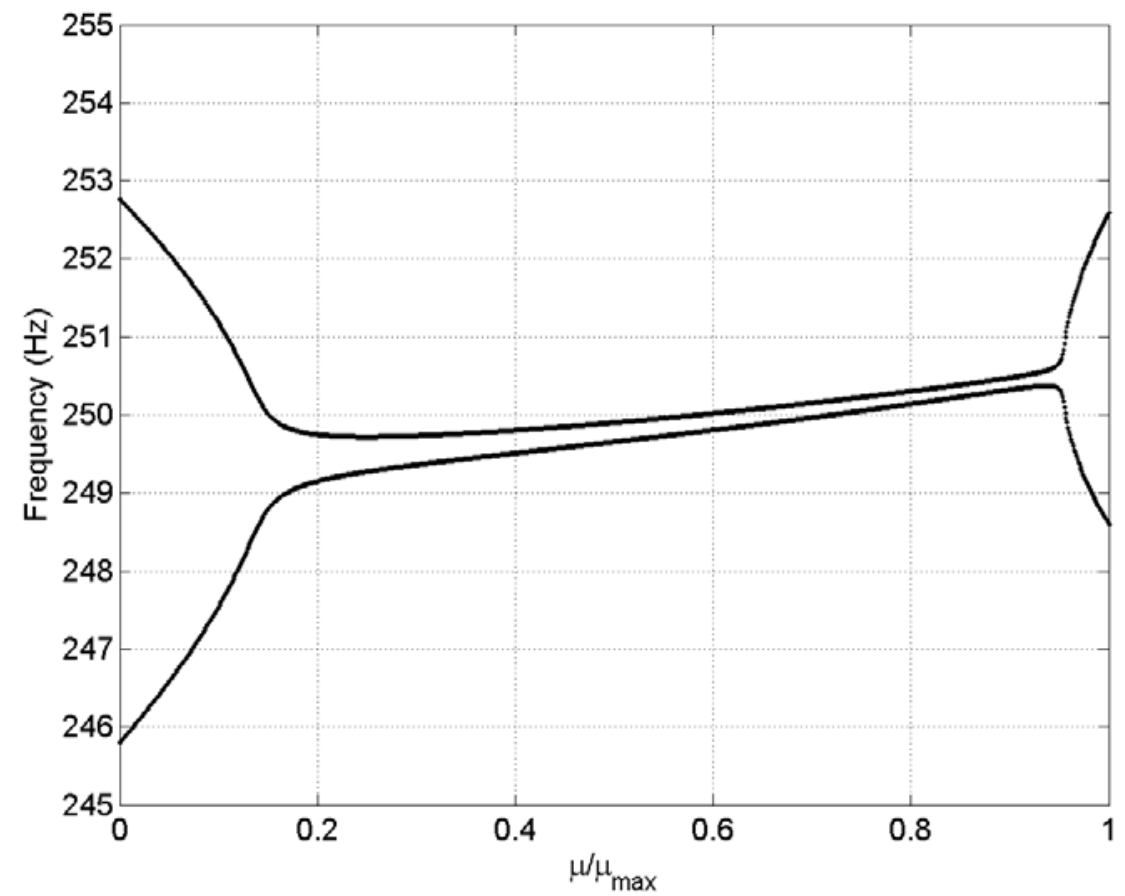

Figure 4 : evolution of the imaginary part for the unstable mode (coalescence of two modes)

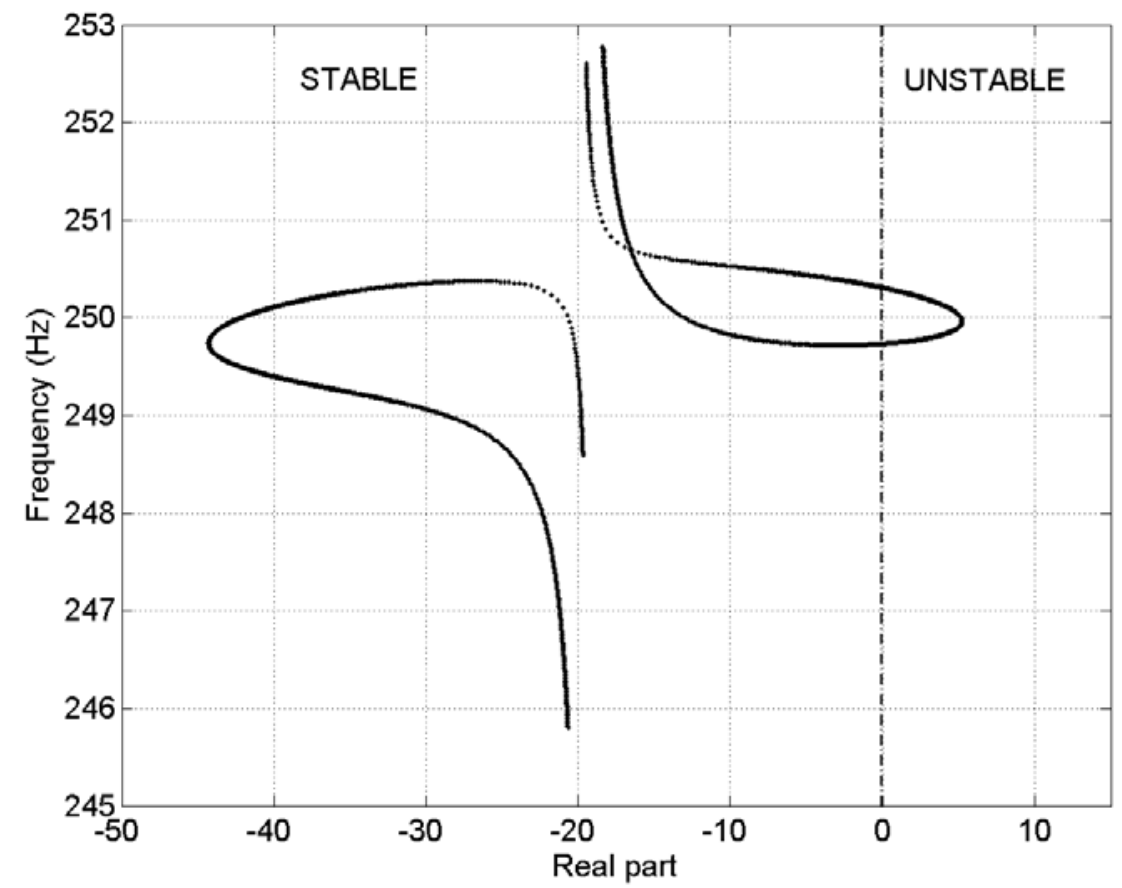

Figure 5 : evolution of the stable and unstable coupled mode in the complex plane 

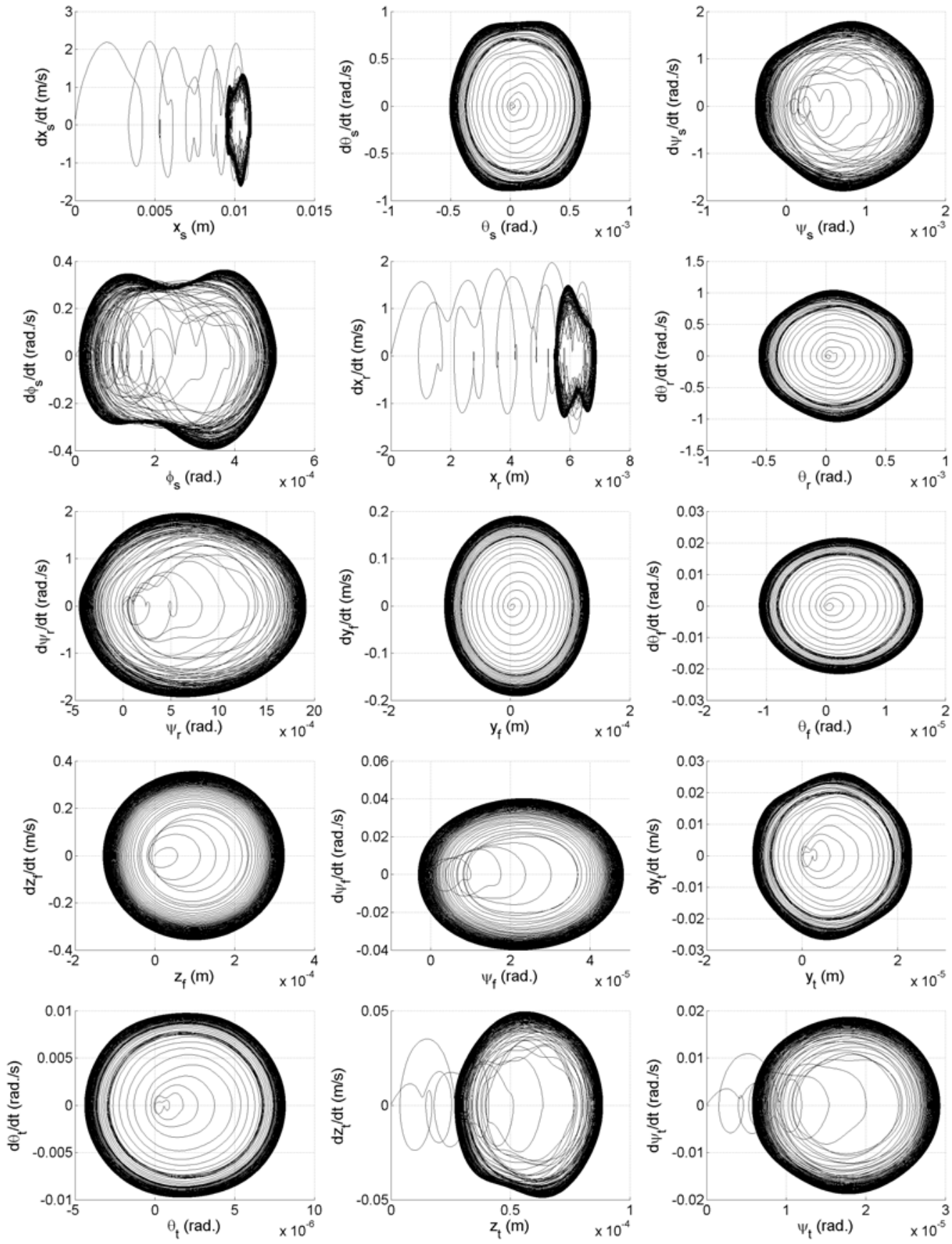

Figure 6 : evolution of the non-linear vibrations for the complete non-linear system by using the $4^{\text {th }}$ order Runge-Kutta algorithm (with $\mu=1.1 \mu_{0}$ and $\mathrm{P} / \mathrm{P}_{\max }=0.5$ ). 


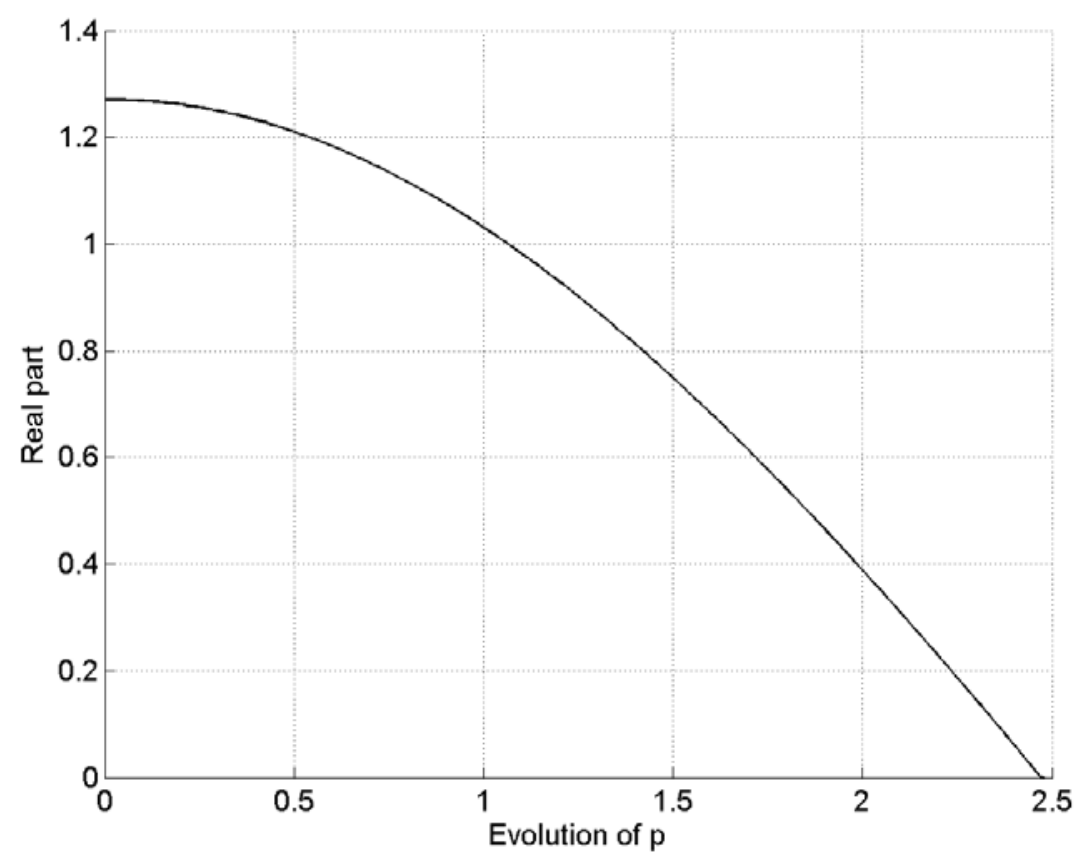

Figure 7: evolution of the real part of the unstable mode via the CNLMA procedure (with $\mu=1.1 \mu_{0}$ and $\mathrm{P} / \mathrm{P}_{\max }=0.5$ ).

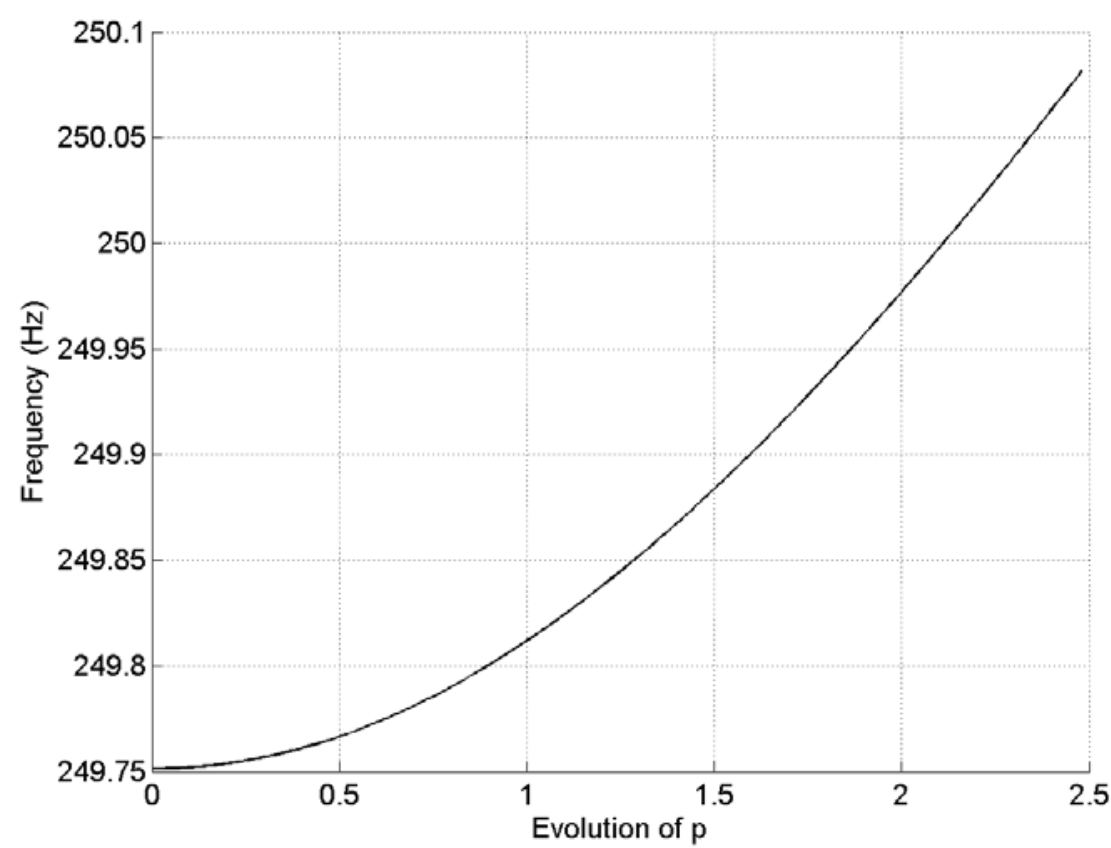

Figure 8 : evolution of the frequency of the unstable mode via the CNLMA procedure (with $\mu=1.1 \mu_{0}$ and $\mathrm{P} / \mathrm{P}_{\max }=0.5$ ). 

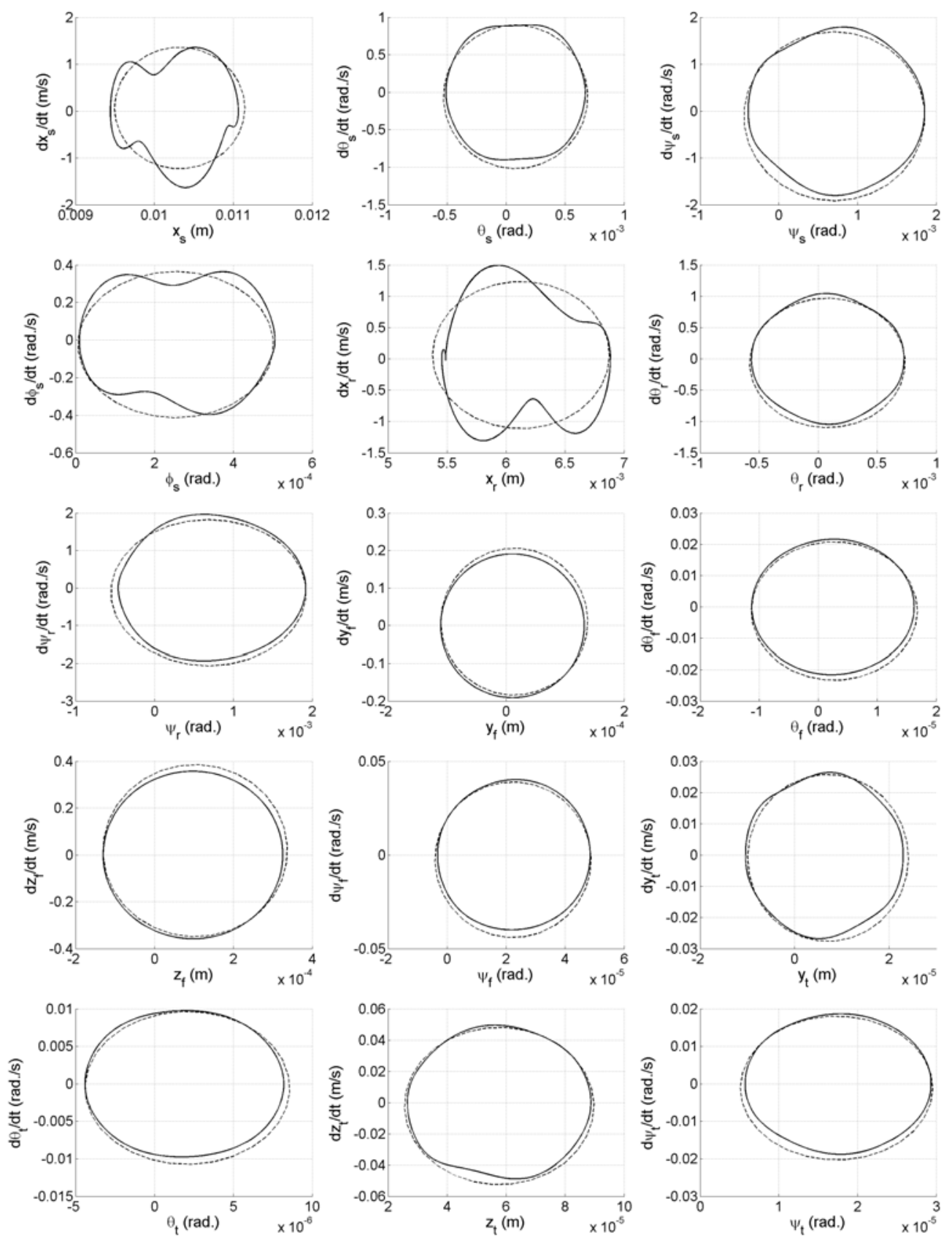

Figure 9 : comparison between the stationary periodic apprximate solution via the CNLMA procedure and the exact non-linear solution for each degree-of-freedom (with $\mu=1.1 \mu_{0}$ and $\mathrm{P} / \mathrm{P}_{\max }=0.5$ ). 


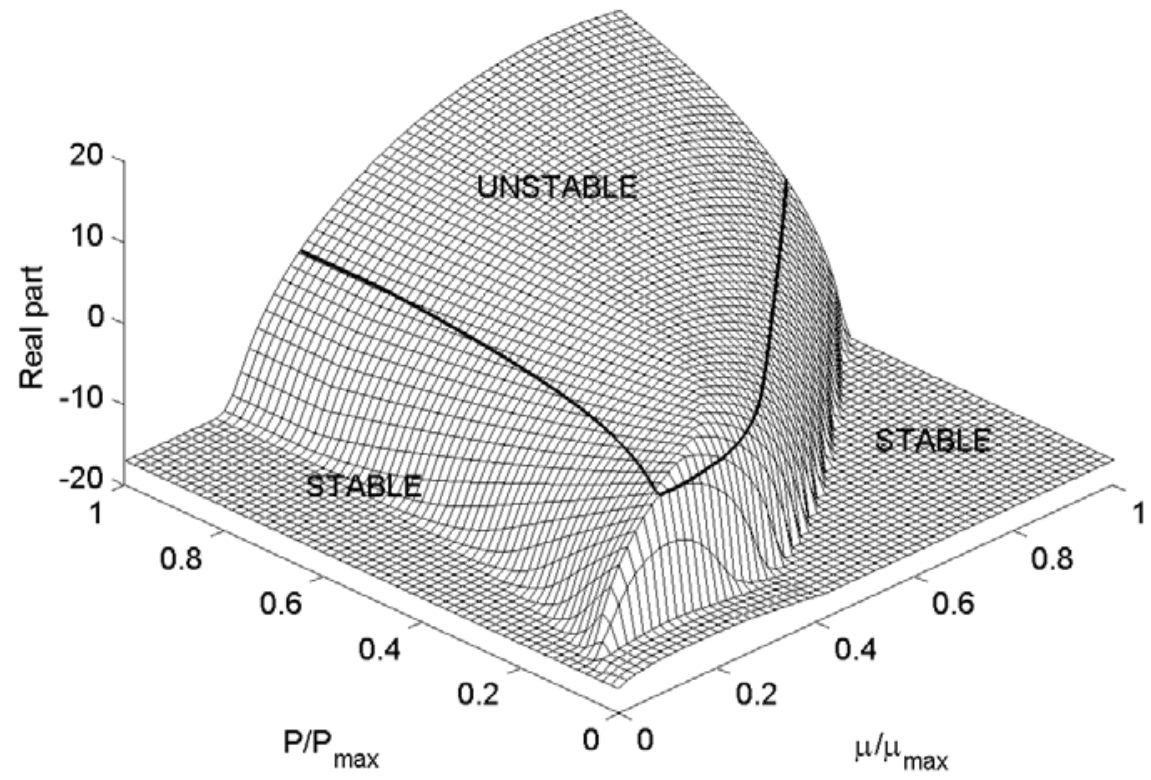

Figure 10 : evolution of the real part for the stable-unstable mode versus the pressure and the friction coefficient

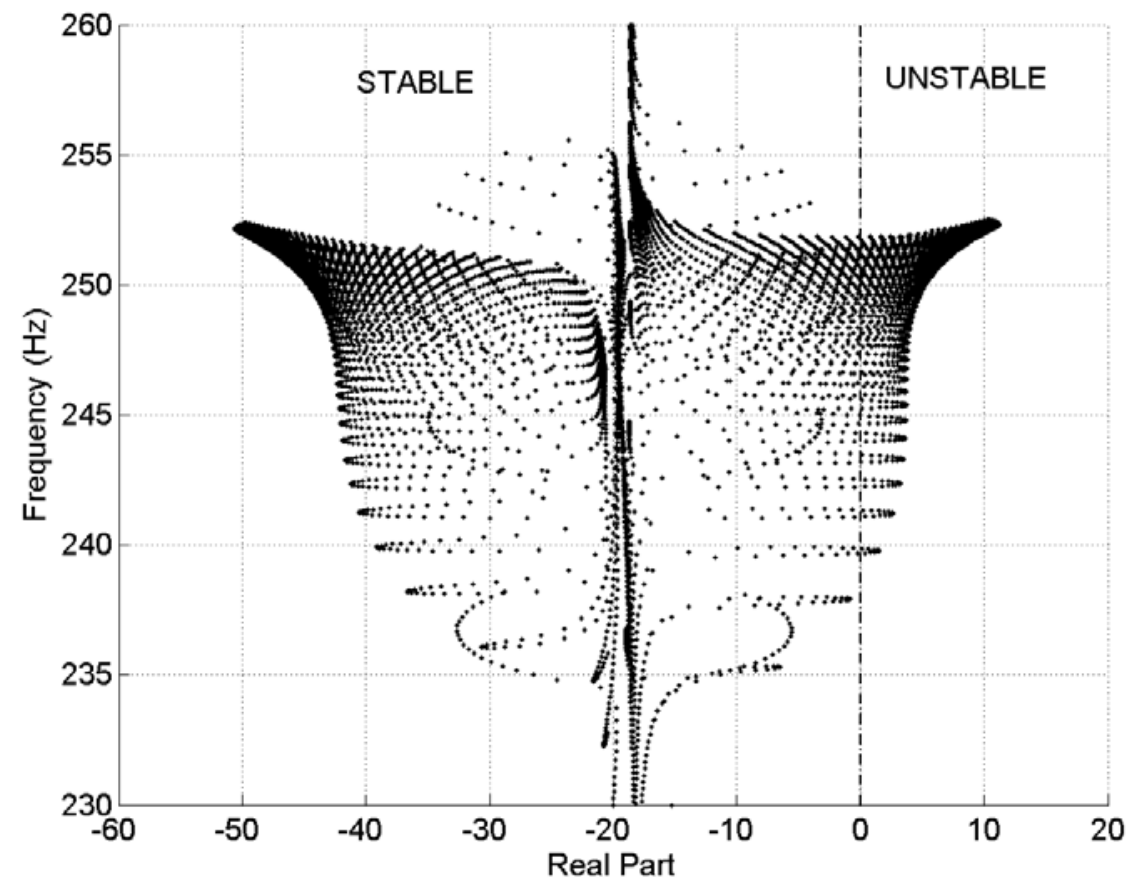

Figure 11 : evolution of the imaginary and real part for the stable-unstable mode versus the pressure and the friction coefficient in the complexe plane 


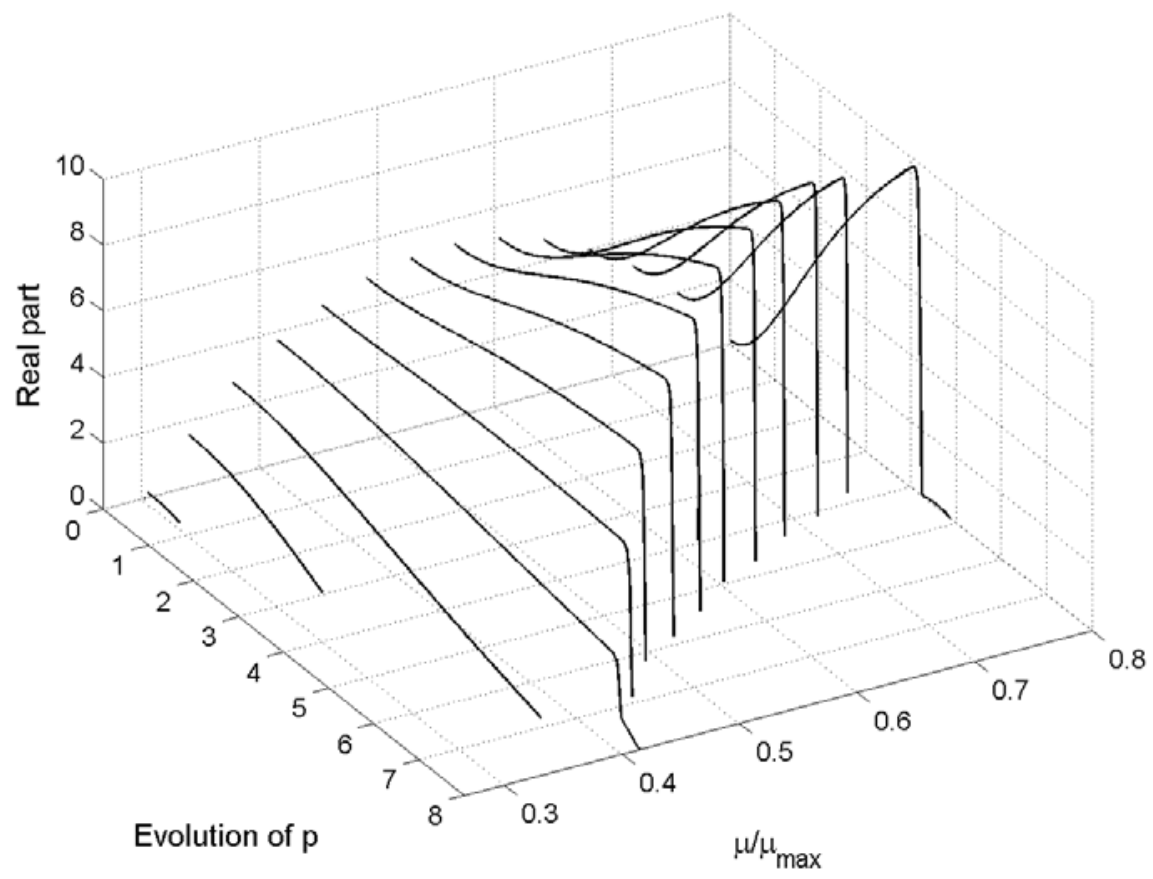

Figure 12 : evolution of the real part for the unstable mode versus the friction coefficient (with $\mathrm{P} / \mathrm{P}_{\max }=0.5$ ). 

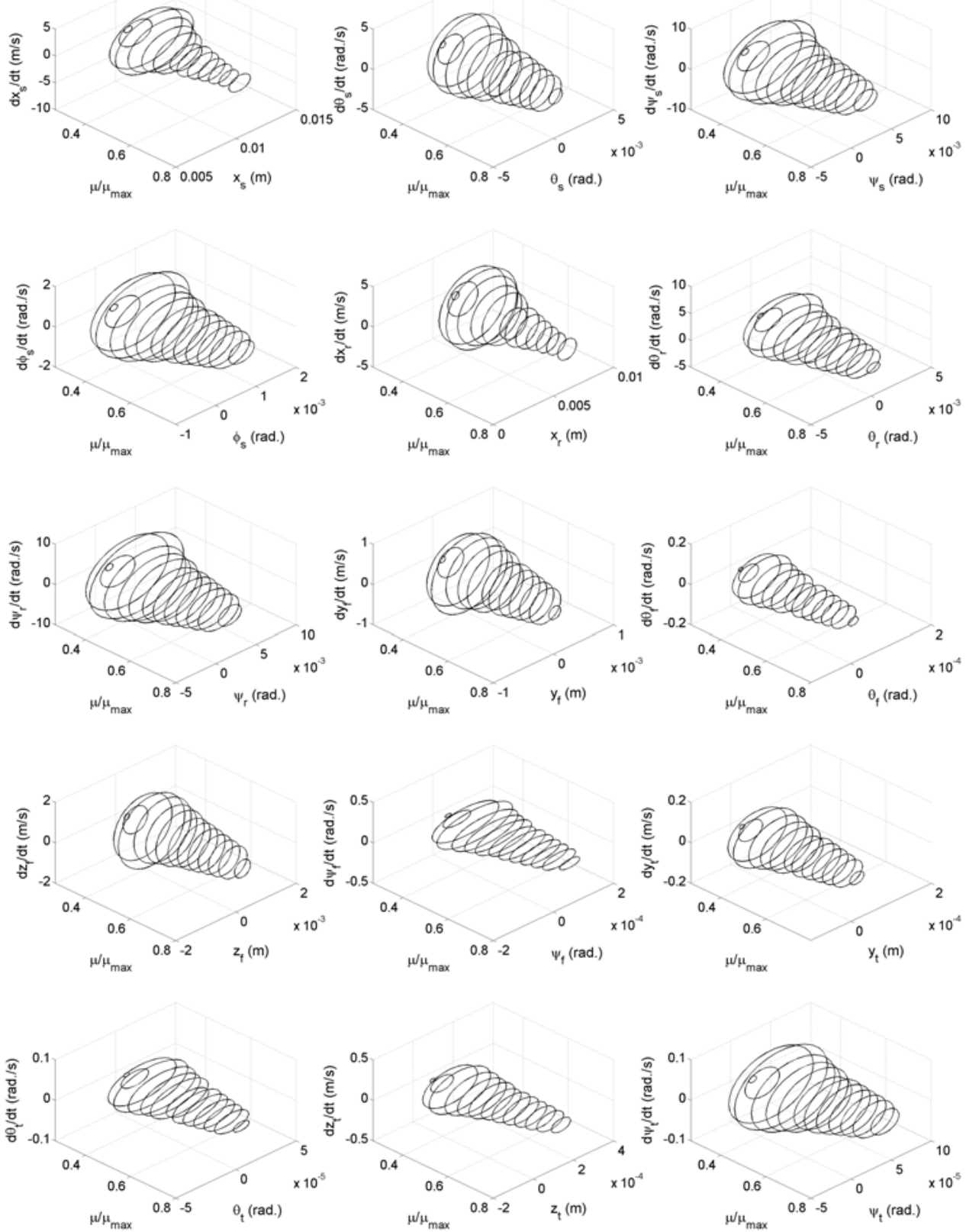

Figure 13 : evolutions of limit cycle amplitudes versus the friction coefficient (with

$$
\mathrm{P} / \mathrm{P}_{\max }=0.5 \text { ). }
$$



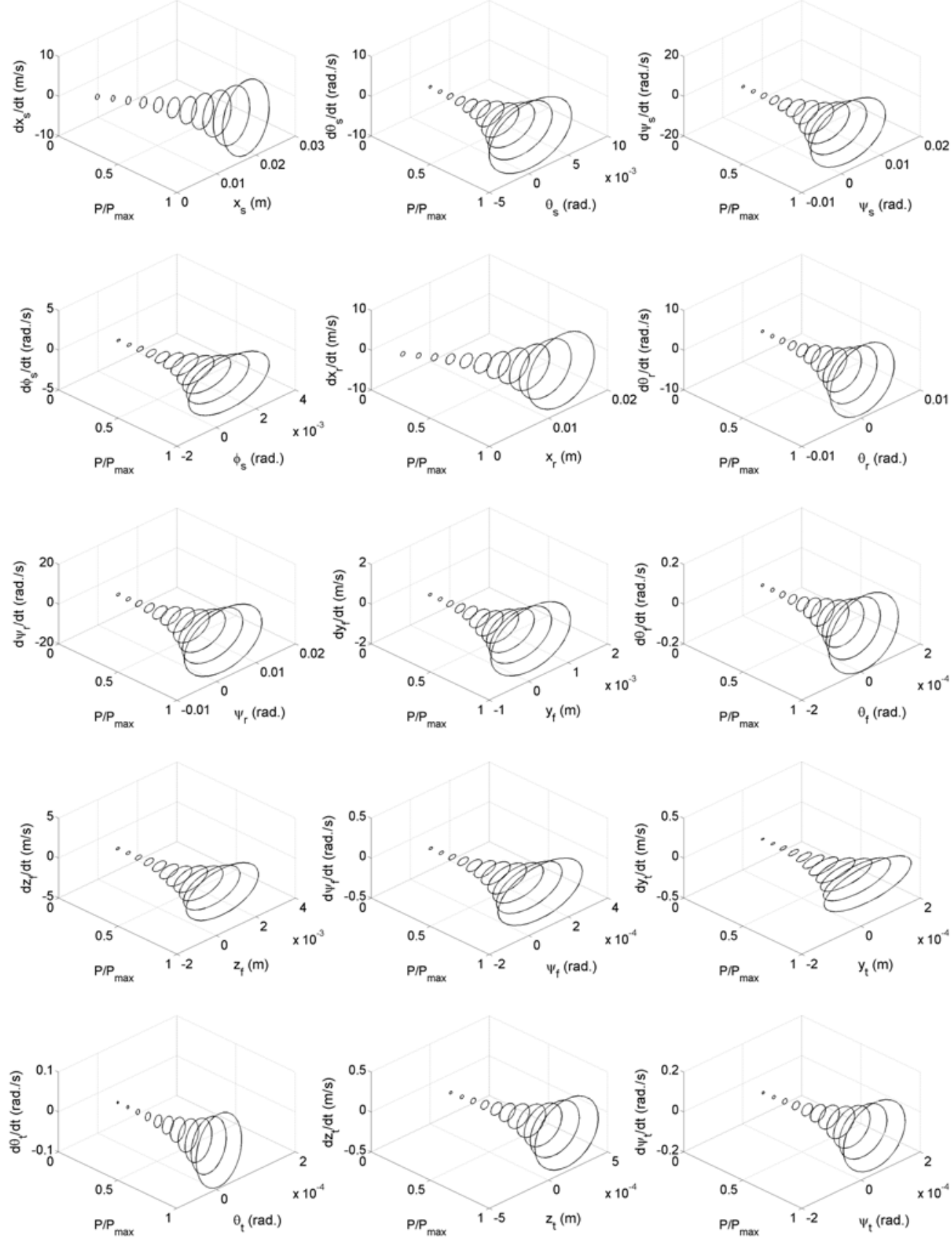

Figure 14 : evolutions of limit cycle amplitudes versus the brake pressure (with $\mu=1.1 \mu_{0}$ ) 


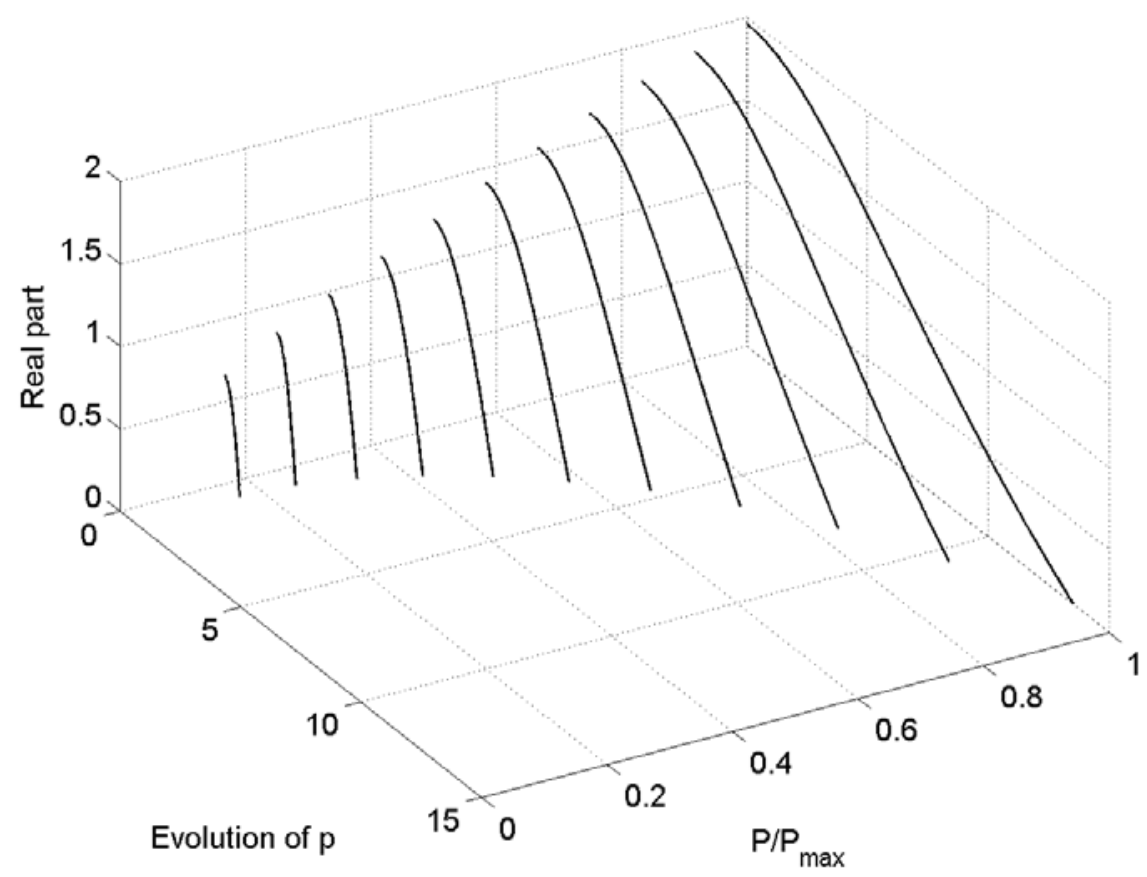

Figure 15 : evolution of the real part for the unstable mode versus the brake presure (with $\left.\mu=1.1 \mu_{0}\right)$
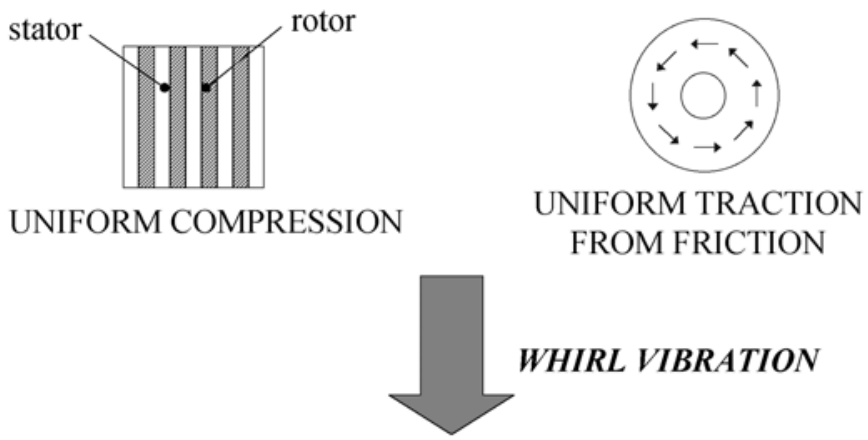
FROM FRICTION

Increased compression

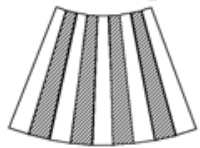

Relaxed compression

WHIRL VIBRATION

ACCORDION DEFORMATION

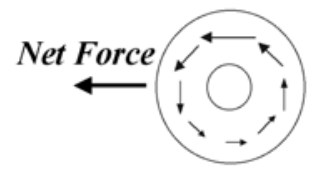

NET FORCE FROM

ACCORDION DEFORMATION

Figure 16: accordeon deformation due to whirl vibration 


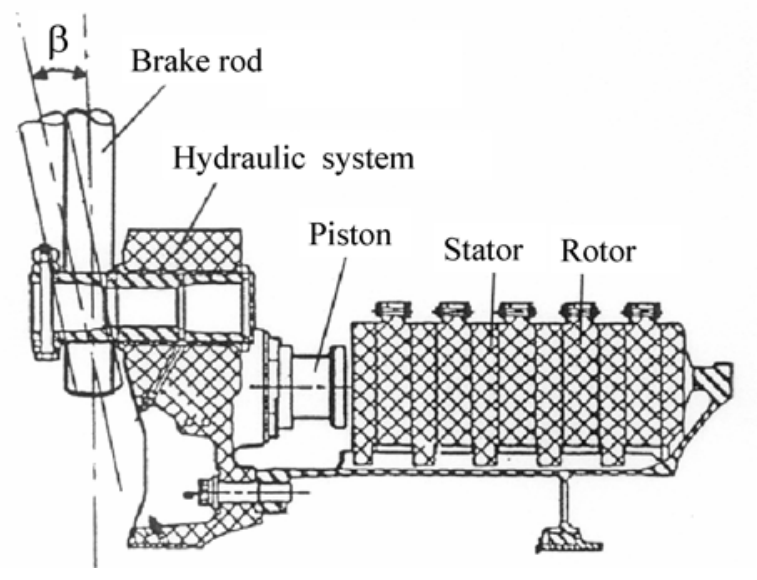

Figure 17: sprag-slip angle $\beta$ induced whirl instability

Friction surface
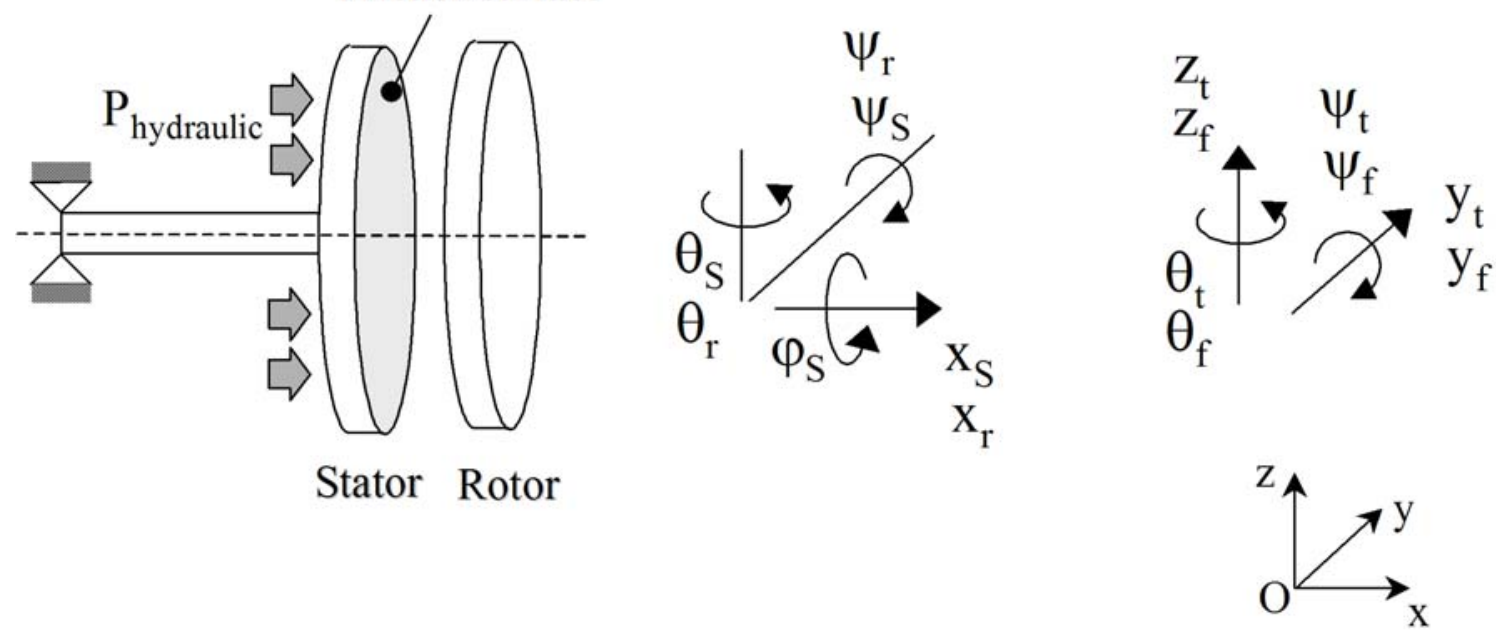

Figure 18: whirl modeling for an aircraft brake system 